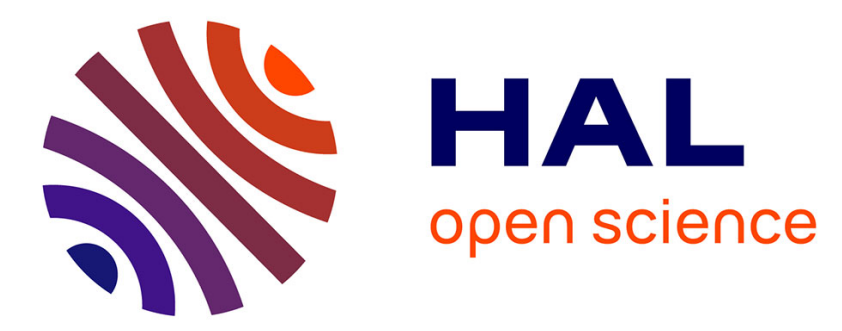

\title{
Finite-horizon discounted optimal control: stability and performance
}

Mathieu Granzotto, Romain Postoyan, Lucian Busoniu, Dragan Nešić, Jamal Daafouz

\section{- To cite this version:}

Mathieu Granzotto, Romain Postoyan, Lucian Busoniu, Dragan Nešić, Jamal Daafouz. Finite-horizon discounted optimal control: stability and performance. IEEE Transactions on Automatic Control, 2021, 66 (2), pp.550-565. 10.1109/TAC.2020.2985904 . hal-02541326

\section{HAL Id: hal-02541326 \\ https://hal.science/hal-02541326}

Submitted on 13 Apr 2020

HAL is a multi-disciplinary open access archive for the deposit and dissemination of scientific research documents, whether they are published or not. The documents may come from teaching and research institutions in France or abroad, or from public or private research centers.
L'archive ouverte pluridisciplinaire HAL, est destinée au dépôt et à la diffusion de documents scientifiques de niveau recherche, publiés ou non, émanant des établissements d'enseignement et de recherche français ou étrangers, des laboratoires publics ou privés. 


\title{
Finite-horizon discounted optimal control: stability and performance
}

\author{
Mathieu Granzotto, Romain Postoyan, Lucian Buşoniu, Dragan Nešić, and Jamal Daafouz
}

\begin{abstract}
Motivated by (approximate) dynamic programming and model predictive control problems, we analyse the stability of deterministic nonlinear discrete-time systems whose inputs minimize a discounted finite-horizon cost. We assume that the system satisfies stabilizability and detectability properties with respect to the stage cost. Then, a Lyapunov function for the closedloop system is constructed and a uniform semiglobal stability property is ensured, where the adjustable parameters are both the discount factor and the horizon length, which corresponds to the number of iterations for dynamic programming algorithms like value iteration. Stronger stability properties such as global exponential stability are also provided by strengthening the initial assumptions. We give bounds on the discount factor and the horizon length under which stability holds and we provide conditions under which these are less conservative than the bounds of the literature for discounted infinite-horizon cost and undiscounted finite-horizon costs, respectively. In addition, we provide new relationships between the optimal value functions of the discounted, undiscounted, infinite-horizon and finite-horizon costs respectively, which are very different from those available in the approximate dynamic programming literature. These relationships rely on assumptions that are more likely to be satisfied in a control context. Finally, we investigate stability when only a near-optimal sequence of inputs for the discounted finitehorizon cost is available, covering approximate value iteration as a particular case.
\end{abstract}

\section{INTRODUCTION}

Finding an optimal sequence of inputs for a dynamical system is highly desirable, finding one that it is also stabilizing is even more so for safety and reliability reasons. For the linear quadratic regulator problem, we know since the 60 's that the optimal feedback law ensures the global exponential stability of the origin of the closed loop system under stabilizability and detectability conditions [13]. Similar results have been derived for nonlinear systems with general infinite-horizon costs, e.g. [15], however such costs are often impossible to minimize exactly. In this paper, we consider general discretetime nonlinear systems where the inputs minimizes a finitehorizon discounted cost in a receding-horizon fashion. By discounted cost, we mean that the stage cost is weighted by a time-varying decaying term $\gamma^{k}$ where $\gamma \in(0,1)$ and $k$ is the discrete time index. Our primary goal is to identify conditions under which the induced closed-loop system exhibits stability properties.

M. Granzotto, R. Postoyan and J. Daafouz are with the Université de Lorraine, CNRS, CRAN, F-54000 Nancy, France (e-mails: \{name.surname\}@univlorraine.fr).

L. Buşoniu is with the Department of Automation, Technical University of Cluj-Napoca, Romania (e-mail: lucian.busoniu@ aut.utcluj.ro).

D. Nešić is with the Department of Electrical and Electronic Engineering, University of Melbourne, Parkville, VIC 3010, Australia (e-mail: dnesic@unimelb.edu.au).
Finite-horizon discounted costs are important for several reasons. Various algorithms used in approximate dynamic programming generate near-optimal control inputs for nonlinear discrete-time systems, see e.g., [3,11,19,23,25]. In most cases, the cost is discounted to obtain a contraction property of the cost brought by the discount factor $\gamma$, which is the cornerstone of numerous studies. A closer look at value iteration, which is one of the pillars of dynamic programming, reveals that the algorithm stops after $d$ iterations, estimating a value corresponding to the sum of $d$ discounted stage costs. In other words, even though value iteration aims for the infinite-horizon solution, when initialized with zero values and stopped after a number of iterations, the algorithm actually provides a horizon$d$ discounted optimal solution. This feature is shared by other methods that work similarly, such as optimistic planning [11]. On the other hand, finite-horizon costs are also relevant in their own right when we want to give to the stage cost more importance in close future and less as time grows, as in model predictive control for instance; see [9,17] for applications on smart buildings and economy, respectively.

As far as we are aware of, all the results of the literature, which consider finite-horizon discounted costs elude the question of stability. In particular, the field of (approximate) dynamic programming largely concentrates on optimality, leaving the question of the stability of the induced closed-loop systems open. To endow (approximate) dynamic programming algorithms with stability guarantees is an important topic as it will bring closer the use of such algorithms for safety-critical applications, and will help build a bridge between techniques from approximate dynamic programming and reinforcement learning on the one hand, and control on the other. In the context of this paper, the challenge is to cope with the discount factor $\gamma$ as well as the fact that cost function has a finitehorizon. Stability results for finite-horizon undiscounted costs are abundant in the model predictive control literature e.g., $[6,7,15,24]$. Yet, when the cost is both discounted and has finite-horizon, no results are available regarding the stability of the closed-loop system, as far as we know. In [20], the authors investigate dissipativity properties of finite-horizon discounted costs, but not stability. On the other hand, the work in [21] (and recently [4]) provides results on the stability of nonlinear discrete-time systems controlled by an optimal sequence of inputs, which minimizes an infinite-horizon discounted cost. The fact that the cost is finite-horizon and involves a discount factor leads to major technical difficulties in the stability analysis. Indeed, there is an intricate interaction between the horizon and the discount factor, and stating a precise relationship under which stability holds is non-trivial. In other 
words, we cannot simply combine the results of, [6] and [21] to obtain the desired result: a new stability analysis is needed. It can be noted that dynamic programming tools are usually seen in a stochastic setting [1], for which relationships between infinite-horizon discounted and finite-horizon undiscounted problems can be established [18]. In this paper, we concentrate on the deterministic setting, for which such links do not apply. Nevertheless, we believe that the presented approach could serve as a basis to address the problem for stochastic processes in the future.

We assume that the inputs are computed in a recedinghorizon fashion, as in model predictive control. This would correspond to applying the first element of the input sequence obtained after $d$ iterations of the value iteration algorithm as state feedback. We start with essentially the same assumptions as in [6,21], namely that the plant is stabilizable and the stage cost is detectable. As in [6], we use a generic measure $\sigma$ to define stability, thus covering the stability of the origin or of more general sets in a unified way. We then present a new Lyapunov analysis, which allows us to prove that the closed-loop system satisfies a semiglobal practical stability property provided the horizon length $d$ and the discount factor $\gamma$ are sufficiently large and sufficiently close to 1 , respectively. This analysis is one of the main contributions of the paper. Stronger stability properties are then provided by strengthening the assumptions, and explicit bounds on $\gamma$ and $d$ are given. The Lyapunov function used to prove stability is continuous under mild assumptions, ensuring the robustness of the stability property according to [16]. It appears that the presented Lyapunov analysis differs from those in $[6,21]$ even when $\gamma=1$ and $d=\infty$, respectively. This allows us to derive new bounds on $\gamma$ or $d$, according to the considered scenario, which may be less conservative than those in $[6,21]$ as shown on several examples in the preliminary version of this work [5]; this constitutes an additional contribution of the paper.

Our assumptions also allows improving the performance analysis currently available in the corresponding literature, which is of major interest. As explained above, the minimization of the discounted finite-horizon cost may be done to approximately optimize the original discounted infinite-horizon cost. The bounds on the difference between these two costs provided in approximate dynamic programming are of the form $\frac{\gamma^{d}}{1-\gamma}$, see [1]. This holds even when the solution is exactly represented. We provide very different bounds here, which have the following key features: (i) they do not explode to infinity as $\gamma$ tends to 1 ; (ii) they are small when $\sigma(x)$ is small, where $x$ is the plant initial condition and $\sigma$ the measure we use to state stability. Furthermore, these bounds do not require the stage cost to take values in $[0,1]$, as in [1]. We believe that there is an important message here. Related results are derived in [8] for undiscounted costs. Beside the fact that we allow the cost to be discounted here, we rely on different assumptions compared to [8], which leads to different bounds.

Finally, we study the scenario where the sequence of inputs is only near-optimal. The assumption we make on the inputs covers those generated by approximate value iteration as a particular case. We then adapt the arguments used before to ensure the stability of the closed-loop system, still in a semiglobal and practical sense. Compared to the related results of the literature, [10] in particular: (i) we study stability of a generic closed set, and not only of the origin; (ii) we do not need the explicit knowledge of a globally stabilizing policy; (iii) we address discounted costs; (iv) the stage cost is not necessarily quadratic.

Compared to the preliminary version of this work in [5], the completely novel elements are: the performance analysis, i.e. the relationship between the discounted finite-horizon cost and the infinite-horizon one, and the case of near-optimal inputs. Additionally, we provide conditions for semiglobal asymptotic stability as well as the full proofs of all results.

The rest of the paper is organized as follows. The problem is formally stated in Section II. The main results are given in Section III. The relationships between the optimal value functions of the discounted/undiscounted, finite/infinite-horizon costs are provided in Section IV. In Section V, we investigate the case where the sequence of inputs is near-optimal. The proofs are presented in Section VI and conclusions are given in Section VII. The appendix contains technical results needed in the main proofs.

Notation. Let $\mathbb{R}:=(-\infty, \infty), \mathbb{R}_{\geq 0}:=[0, \infty), \mathbb{Z}_{\geq 0}:=$ $\{0,1,2, \ldots\}$ and $\mathbb{Z}_{>0}:=\{1,2, \ldots\}$. We use $(x, y)$ to denote $\left[x^{T}, y^{T}\right]^{T}$, where $(x, y) \in \mathbb{R}^{n} \times \mathbb{R}^{m}$ and $n, m \in \mathbb{Z}_{>0}$. A function $\chi: \mathbb{R}_{\geq 0} \rightarrow \mathbb{R}_{\geq 0}$ is of class $\mathcal{K}$ if it is continuous, zero at zero and strictly increasing, and it is of class $\mathcal{K}_{\infty}$ if it is of class $\mathcal{K}$ and unbounded. A continuous function $\beta: \mathbb{R}_{\geq 0} \times \mathbb{R}_{\geq 0} \rightarrow \mathbb{R}_{\geq 0}$ is of class $\mathcal{K} \mathcal{L}$ when $\beta(\cdot, t)$ is of class $\mathcal{K}$ for any $t \geq 0$ and $\beta(s, \cdot)$ is decreasing to 0 for any $s \geq 0$. The notation $\mathbb{I}$ stands for the identity map from $\mathbb{R}_{\geq 0}$ to $\mathbb{R}_{\geq 0}$. For any sequence $\boldsymbol{u}=\left[u_{0}, u_{1}, \ldots\right]$ of length $d \in \mathbb{Z}_{\geq 0} \cup\{\infty\}$ where $u_{i} \in \mathbb{R}^{m}, i \in\{0, \ldots, d\}$, and any $k \in\{0, \ldots, d\}$, we use $\left.\boldsymbol{u}\right|_{k}$ to denote the first $k$ elements of $\boldsymbol{u}$, i.e. $\left.\boldsymbol{u}\right|_{k}=\left[u_{0}, \ldots, u_{k-1}\right]$ and $\left.\boldsymbol{u}\right|_{0}=\varnothing$ by convention. Let $f: \mathbb{R} \rightarrow \mathbb{R}$, we use $f^{(k)}$ for the composition of function $f$ to itself $k$ times, where $k \in \mathbb{Z}_{>0}$, and $f^{(0)}=\mathbb{I}$. We use $\lfloor\cdot\rfloor$ to denote the floor function. The Euclidean norm of a vector $x \in \mathbb{R}^{n}$ is denoted by $|x|$.

\section{Problem Statement}

Consider the system

$$
x_{k+1}=f\left(x_{k}, u_{k}\right),
$$

with state $x \in \mathbb{R}^{n}$, input $u \in \mathcal{U}(x) \subseteq \mathbb{R}^{m}$, where $\mathcal{U}(x)$ is the nonempty set of admissible inputs for state $x$, and $f: \mathcal{W} \rightarrow \mathbb{R}^{n}$ where $\mathcal{W}:=\left\{(x, u): x \in \mathbb{R}^{n}, u \in\right.$ $\mathcal{U}(x)\}$. We use $\phi\left(k, x,\left.\boldsymbol{u}\right|_{k}\right)$ to denote the solution to system (1) at time $k \in \mathbb{Z}_{\geq 0}$ with initial condition $x$ and inputs $\left.\boldsymbol{u}\right|_{k}=\left[u_{0}, u_{1}, \ldots, u_{k-1}\right]$, with the convention $\phi(0, x, \cdot)=$ $\phi(0, x, \varnothing)=x$.

We study discounted finite-horizon costs of the form

$$
J_{\gamma, d}(x, \boldsymbol{u}):=\sum_{k=0}^{d} \gamma^{k} \ell\left(\phi\left(k, x,\left.\boldsymbol{u}\right|_{k}\right), u_{k}\right)
$$

where $x \in \mathbb{R}^{n}, \boldsymbol{u}$ is a sequence of $d+1$ admissible inputs, $\ell: \mathcal{W} \rightarrow \mathbb{R}_{\geq 0}, \gamma \in(0,1]$ is the discount factor and $d \in$ $\mathbb{Z}_{>0} \cup\{\infty\}$ is the horizon. 
We assume that for any $x \in \mathbb{R}^{n}, \gamma \in(0,1]$ and $d \in$ $\mathbb{Z}_{>0} \cup\{\infty\}$, there is a sequence $\boldsymbol{u}$ that minimizes cost (2), as formalized next.

Standing Assumption (SA): For any $x \in \mathbb{R}^{n}, \gamma \in(0,1]$ and $d \in \mathbb{Z}_{>0} \cup\{\infty\}$, there exists a sequence of $d+1$ admissible inputs $\boldsymbol{u}_{\gamma, d}^{*}(x)$, called optimal input sequence, which minimizes (2), i.e.

$$
J_{\gamma, d}\left(x, \boldsymbol{u}_{\gamma, d}^{*}(x)\right)=\min _{\boldsymbol{u}} J_{\gamma, d}(x, \boldsymbol{u})=: V_{\gamma, d}(x),
$$

where $V_{\gamma, d}$ is the optimal cost function.

Conditions to ensure the satisfaction of SA can be found in [14]. According to SA, for any $x \in \mathbb{R}^{n}, \gamma \in(0,1]$ and $d \in \mathbb{Z}_{>0} \cup\{\infty\}$, the following set is non-empty

$$
\begin{gathered}
\mathcal{U}_{\gamma, d}^{*}(x):=\left\{u_{0}: \exists u_{1}, \ldots, u_{d} \in \mathbb{R}^{m}\right. \text { admissible such that } \\
\left.V_{\gamma, d}(x)=J_{\gamma, d}\left(x,\left[u_{0}, \ldots, u_{d}\right]\right)\right\} .
\end{gathered}
$$

Note that $\mathcal{U}_{\gamma, d}^{*}(x)$ may be a set with multiple elements because the optimal sequence may be non-unique for given $x$, $\gamma$ and $d$.

We consider the scenario where system (1) is controlled in a receding horizon fashion in the sense that, at each time instant $k \in \mathbb{Z}_{\geq 0}$, the first element of the optimal sequence $\boldsymbol{u}_{\gamma, d}^{*}\left(x_{k}\right)$, which may be non-unique, is applied to system (1). This leads to the difference inclusion

$$
x_{k+1} \in f\left(x_{k}, \mathcal{U}_{\gamma, d}^{*}\left(x_{k}\right)\right)=: F_{\gamma, d}^{*}\left(x_{k}\right),
$$

where $f\left(x, \mathcal{U}_{\gamma, d}^{*}(x)\right)$ is the set $\left\{f(x, u): u \in \mathcal{U}_{\gamma, d}^{*}(x)\right\}$. We denote by $\phi(k, x)$, with some abuse of notation, a solution to (5) at time $k \in \mathbb{Z}_{\geq 0}$ with initial condition $x \in \mathbb{R}^{n}$.

Our main objective is to analyse the stability and robustness of system (5) using Lyapunov-based arguments. In particular, we want to investigate the influence of the cost parameters $\gamma$ and $d$ on stability. To this end, we make the following assumptions, inspired by [6], on the stabilizability and detectability of system (1) and cost function (2).

Assumption 1: There exist $\bar{\alpha}_{V}, \alpha_{W} \in \mathcal{K}_{\infty}$, continuous functions $W, \sigma: \mathbb{R}^{n} \rightarrow \mathbb{R}_{\geq 0}, \bar{\alpha}_{W}: \mathbb{R}_{\geq 0} \rightarrow \mathbb{R}_{\geq 0}$ continuous, non-decreasing and zero at zero, such that the following conditions hold.

(i) For any $x \in \mathbb{R}^{n}, \gamma \in(0,1]$ and $d \in \mathbb{Z}_{>0} \cup\{\infty\}$,

$$
V_{\gamma, d}(x) \leq \bar{\alpha}_{V}(\sigma(x)) .
$$

(ii) For any $x \in \mathbb{R}^{n}, u \in \mathcal{U}(x)$,

$$
\begin{aligned}
W(x) & \leq \bar{\alpha}_{W}(\sigma(x)) \\
W(f(x, u))-W(x) & \leq-\alpha_{W}(\sigma(x))+\ell(x, u) .
\end{aligned}
$$

Function $\sigma$ in Assumption 1 serves as a measure of the distance of the state to the attractor and will be used to define stability. When investigating the stability of the origin for instance, we typically take $\sigma(x)=|x|, \sigma(x)=|x|^{2}$, or $\sigma(x)=$ $x^{T} P x$ with $P$ a real symmetric, positive definite matrix, for any $x \in \mathbb{R}^{n}$. When interested in stability of a non-empty set $\mathcal{A} \subseteq \mathbb{R}^{n}, \sigma$ can be defined as $\sigma=|\cdot|_{\mathcal{A}}$ for instance, where $|x|_{\mathcal{A}}=\inf \{|z-x|: z \in \mathcal{A}\}$ for any $x \in \mathbb{R}^{n}$. Item (i) of Assumption 1 is related to the asymptotic controllability (stabilizability) of system (1) with respect to $\sigma$, see for more detail Section III in [6] and Lemma 1 in [21]. Item (ii) of Assumption 1 is a detectability property of the stage cost $\ell$ with respect to $\sigma$. To see this, consider the particular case where $W=0$ so that (8) reduces to $\alpha_{W}(\sigma(x)) \leq \ell(x, u)$. Thus, when $\ell(x, u)=0, \sigma(x)=0$ since $\alpha_{W} \in \mathcal{K}_{\infty}$.

Remark 1: A more general detectability assumption is made in [6,21], namely $W(f(x, u))-W(x) \leq-\alpha_{W}(\sigma(x))+$ $\chi(\ell(x, u))$ where $\chi \in \mathcal{K}_{\infty}$, instead of (8). It is possible to obtain stability results in this case, at the price of more technicalities. We have not addressed this case in the paper to avoid compromising the clarity of our main results with the technicalities involved in deriving this more general case.

Remark 2: Throughout the text, we assume $\mathbb{I}-\alpha_{W} \circ\left(\bar{\alpha}_{V}+\right.$ $\left.\bar{\alpha}_{W}\right)^{-1} \in \mathcal{K}_{\infty}$. This is without loss of generality, as, if it is not the case, we can always upper-bound $\mathbb{I}-\alpha_{W} \circ\left(\bar{\alpha}_{V}+\bar{\alpha}_{W}\right)^{-1}$ by $\mathbb{I}-\tilde{\alpha}$, which is of class $\mathcal{K}_{\infty}$ for some suitable $\tilde{\alpha} \in \mathcal{K}_{\infty}$, according to Lemma B.1 in [12]. This substitution is enough for the forthcoming stability analysis.

\section{Stability Results}

\section{A. Lyapunov Properties}

The satisfaction of Assumption 1 allows us to derive the following Lyapunov properties, that we use to derive the main stability result for system (5) afterwards.

Theorem 1: Suppose Assumption 1 holds. There exist $\underline{\alpha}_{Y}, \bar{\alpha}_{Y}, \alpha_{Y} \in \mathcal{K}_{\infty}$ and, for any $\gamma \in(0,1]$ and $d \in \mathbb{Z}_{>0} \cup\{\infty\}$, there exists $Y_{\gamma, d}: \mathbb{R}^{n} \rightarrow \mathbb{R}_{\geq 0}$ such that the following holds.

(i) For any $x \in \mathbb{R}^{n}$,

$$
\underline{\alpha}_{Y}(\sigma(x)) \leq Y_{\gamma, d}(x) \leq \bar{\alpha}_{Y}(\sigma(x)) .
$$

(ii) For any $x \in \mathbb{R}^{n}, v \in F_{\gamma, d}^{*}(x)$,

$$
Y_{\gamma, d}(v)-Y_{\gamma, d}(x) \leq \frac{1}{\gamma}\left(-\alpha_{Y}(\sigma(x))+\Upsilon\left(Y_{\gamma, d}(x), \gamma, d\right)\right)
$$

where $\Upsilon: \mathbb{R}_{\geq 0} \times(0,1] \times\left(\mathbb{Z}_{>0} \cup\{\infty\}\right) \rightarrow \mathbb{R}_{\geq 0}$ is defined in Table I, and is such that, for any $s \geq 0$, $\Upsilon(s, \gamma, d) \rightarrow 0$ when $\gamma \rightarrow 1$ and $d \rightarrow \infty$.

Function $Y_{\gamma, d}$ plays the role of a Lyapunov function in Theorem 1, and its expression as well as the expressions of $\alpha_{Y}, \underline{\alpha}_{Y}, \bar{\alpha}_{Y}$ are given in Table I. Item (i) states that it is positive definite and radially unbounded with respect to the set $\{x: \sigma(x)=0\}$, uniformly in $\gamma$ and $d$. Item (ii) of Theorem 1 shows that $Y_{\gamma, d}$ strictly decreases along the solutions to (5) up to a perturbative term $\Upsilon$, which can be made as small as desired by selecting $\gamma$ close to 1 and $d$ big. We stress that $\gamma$ has to be selected close to 1 and $d$ has to be large in order for $\Upsilon$ to be small in (10), which is consistent with previous works on discounted infinite-horizon control [21] and undiscounted finite-horizon control [6] where a similar Lyapunov inequalities are given. Theorem 1 is actually a generalization of Theorem 1 in [6] to discounted cost and of Theorem 1 in [21] to finitehorizon. The perturbative term $\Upsilon$ differs from the corresponding one in (5) in [6] when $\gamma=1$, and from the one in item (b) of Theorem 1 in [21] when $d=\infty$, because of the way the Lyapunov analysis is carried out in the proof of Theorem 1, 
see Section VI. The new analysis we propose is motivated by the fact that it leads to different bounds, on $d$ (and $\gamma$ ) under which stability is preserved. This is discussed in more detail in Section III-D.

\section{B. Main Result}

We are ready to state the main stability result.

Theorem 2: Consider system (5) and suppose Assumption 1 holds. There exists $\beta \in \mathcal{K} \mathcal{L}$ such that for any $\delta, \Delta>0$, there exist $\gamma^{*} \in(0,1)$ and $d^{*} \in \mathbb{Z}_{>0}$ such that for any $\gamma \in\left(\gamma^{*}, 1\right]$, $d \in\left(d^{*}, \infty\right], x \in\left\{z \in \mathbb{R}^{n}: \sigma(z) \leq \Delta\right\}$, any solution $\phi(\cdot, x)$ to system (5) satisfies, for all $k \in \mathbb{Z}_{\geq 0}$

$$
\sigma(\phi(k, x)) \leq \max \{\beta(\sigma(x), k), \delta\}
$$

Theorem 2 ensures a semiglobal practical stability property, i.e. given any set of initial conditions of the form $\left\{z \in \mathbb{R}^{n}\right.$ : $\sigma(z) \leq \Delta\}$ where $\Delta>0$, and any (arbitrarily small) $\delta$, we can select $\gamma$ and $d$ such that (11) holds. The key inequality for deriving $\gamma^{*}$ and $d^{*}$ can be found in the proof of Theorem 2, see (34). A clear relationship between the choice of $\gamma^{*}$ and $d^{*}$ is difficult to draw for Theorem 2. Explicit bounds are provided in the sequel by strengthening the conditions of Theorem 2, which also allows us to ensure stronger stability properties.

Remark 3: Theorem 2 holds even when the horizon $d$ of cost (2) varies with time as long as it remains larger than $d^{*}$ as defined in Theorem 2 . While we omit the proof, this remark implies some flexibility on the horizon, which could be exploited when employing an online optimization scheme.

We know from [16] that it is essential to work with a continuous Lyapunov function to endow the stability properties with some nominal robustness ${ }^{1}$. Here, function $Y_{\gamma, d}$ in Theorem 2 serves as a Lyapunov function. To ensure it is continuous, we need to guarantee that $V_{\gamma, d}$ is continuous, since $Y_{\gamma, d}=V_{\gamma, d}+W$ and $W$ is continuous according to Assumption 1. Additional assumptions are needed for this purpose.

Assumption 2: The following properties hold.

(i) $f$ and $\ell$ are continuous.

(ii) The admissible input set is uniform in $x$, i.e. $\mathcal{U}(x)=\mathcal{U}$ for all $x \in \mathbb{R}^{n}$.

(iii) Either $\mathcal{U}$ is bounded, i.e. there exists a ball of finite radius $\mathcal{B}$ such that $\mathcal{U} \subseteq \mathcal{B}$, or for each compact set $\mathcal{C}$ $\eta \in \mathbb{R}$, and $d \in \mathbb{Z}_{\geq 0}$, there exists $\mu>0$ such that for any $x \in \mathcal{C}$, all admissible sequences of inputs $\boldsymbol{u}$ of length $d+1$ satisfying $J_{\gamma, d}(x, \boldsymbol{u}) \leq \eta$ satisfy $\left|u_{k}\right| \leq \mu$ for $k \in\{0, \ldots, d\}$.

The next lemma ensures the continuity of $V_{\gamma, d}$ when $d$ is finite.

\footnotetext{
${ }^{1}$ As noted in [21], to apply Theorem 2.8 in [16] the set-valued mapping $F_{\gamma, d}^{*}$ in (5) also has to be such that $F_{\gamma, d}^{*}(x)$ is nonempty and compact for any $x \in \mathbb{R}^{n}$. Non-emptiness follows from SA. Compactness of $F_{\gamma, d}^{*}$ proceeds from the compactness of $\mathcal{U}_{\gamma, d}^{*}(x)$ (when $f$ is continuous, which is assumed to be the case in Lemma 1), which is a consequence of the conditions of Lemma 1 and the continuity of $V_{\gamma, d}$ proved in this lemma, according to item (a) of Theorem 1.17 in [22].
}

Lemma 1: Consider system (1) and suppose Assumption 2 holds. For any $\gamma \in(0,1]$ and $d \in \mathbb{Z}_{>0}$, function $V_{\gamma, d}$ is continuous.

When $d$ is infinite, Theorem 3 in [21] provides conditions under which $V_{\gamma, d}$ is continuous.

\section{Stronger Stability Properties}

We first strengthen the conditions of Theorem 2 to ensure a uniform semiglobal asymptotic stability property.

Corollary 1: Suppose that Assumption 1 is satisfied and there exist $L>0, \bar{a}_{W} \geq 0, a_{W}, \bar{a}_{V}>0$ such that $\bar{\alpha}_{V}(s) \leq \bar{a}_{V} \cdot s$, $\bar{\alpha}_{W}(s) \leq \bar{a}_{W} \cdot s, \alpha_{W}(s) \geq a_{W} \cdot s$ for any $s \in[0, L]$. Let $\Delta>0$, and select $\gamma^{*} \in(0,1]$ and $d^{*} \in \mathbb{Z}_{>0}$ such that

$$
\begin{array}{r}
\left(\frac{1-\frac{a_{W}}{\bar{a}_{Y}}}{\gamma^{*}}\right)^{d^{*}} \bar{a}_{Y} L \leq \alpha_{W}(L) \\
1-\gamma^{*}+\frac{\bar{a}_{V}}{a_{W}}\left(1-\frac{a_{W}}{\bar{a}_{Y}}\right)^{d^{*}}<\frac{a_{W}}{\bar{a}_{Y}},
\end{array}
$$

and for any $(\gamma, d) \in\left(\gamma^{*}, 1\right] \times\left(d^{*}, \infty\right]$,

$$
\Upsilon\left(\bar{\alpha}_{Y}(\Delta), \gamma, d\right) \leq\left(1-\frac{\gamma}{2}\right) \widetilde{\alpha}_{Y}\left(\bar{a}_{Y} L\right),
$$

where $\bar{a}_{Y}:=\bar{a}_{V}+\bar{a}_{W}, \widetilde{\alpha}_{Y}:=\alpha_{W} \circ\left(\bar{\alpha}_{V}+\bar{\alpha}_{W}\right)^{-1}$ and $\Upsilon$ is defined in Table I. Then, there exist $\beta \in \mathcal{K} \mathcal{L}$ independent of $\Delta$ such that, for any $x \in\left\{z \in \mathbb{R}^{n}: \sigma(z) \leq \Delta\right\}$, any solution $\phi(\cdot, x)$ to system (5) satisfies, $\sigma(\phi(k, x)) \leq \beta(\sigma(x), k)$ for all $k \in \mathbb{Z}_{\geq 0}$.

Corollary 1 ensures a uniform semiglobal asymptotic stability property for set $\{x: \sigma(x)=0\}$, i.e. given the set of initial conditions $\left\{z \in \mathbb{R}^{n}: \sigma(z) \leq \Delta\right\}$ where $\Delta$ is any fixed strictly positive real number, we can select $\gamma$ and $d$ such that (11) holds with $\delta=0$. Consistently with Theorem 2, to find a suitable pair $\left(\gamma^{*}, d^{*}\right)$, we have to take $\gamma^{*}$ close to 1 and $d^{*}$ large so that (12)-(14) hold.

Remark 4: Compared to Corollary 2 in [6] and Corollary 1 in [21], where similar results are derived for infinitehorizon discounted cost and finite-horizon undiscounted cost respectively, we require inequality (12). This is not a limitation of our analysis, but an omission of [21] and [6]. Indeed, $f(s) \leq \bar{f} s$ and $g(s) \leq \bar{g} s$ for $s \in[0, L]$ where $\bar{f}, \bar{g}>0$, does not imply that $f(g(s)) \leq \bar{f} \bar{g} s$ for $s \in[0, L]$, as was used previously in Corollary 1 of [21] and Corollary 2 of [6]. For a counterexample, consider $f(s):=s^{2}$ and $g(s):=2 s$ for $L=1$. We have $\bar{f}=1, \bar{g}=2$, yet $f(g(s))=4 s^{2}>2 s=\bar{f} \bar{g} s$ for $s \in\left(\frac{1}{2}, 1\right]$ which is a contradiction. Thus, a correct analysis has to verify that every sub-part of the chain of functions is inside a valid range. Fortunately, this does not lead to a loss of generality of Corollary 1, only requiring an extra condition on $\gamma^{*}$ and $d^{*}$, which can always be verified.

We can formulate stronger stability properties, namely uniform global exponential stability, when conditions of Corollary 1 hold with $L=\infty$.

Corollary 2: Suppose that Assumption 1 is satisfied and there exist $\bar{a}_{W} \geq 0, a_{W}, \bar{a}_{V}>0$ such that $\bar{\alpha}_{V}(s) \leq \bar{a}_{V} \cdot s$, $\bar{\alpha}_{W}(s) \leq \bar{a}_{W} \cdot s, \alpha_{W}(s) \geq a_{W} \cdot s$ for any $s \geq 0$. Let $\gamma^{*}, d^{*}$ be such that

$$
1-\gamma^{*}+\frac{\bar{a}_{V}}{a_{W}}\left(1-\frac{a_{W}}{\bar{a}_{V}+\bar{a}_{W}}\right)^{d^{*}}<\frac{a_{W}}{\bar{a}_{V}+\bar{a}_{W}} .
$$


Table I: Expressions for the functions used in Theorem 1

\begin{aligned} & \hline$Y_{\gamma, d}::=V_{\gamma, d}+W \\ & \underline{\alpha}_{Y}:= \alpha_{W} \\ & \bar{\alpha}_{Y}:= \bar{\alpha}_{V}+\bar{\alpha}_{W} \\ & \Upsilon(s, \gamma, d):= \begin{cases}(1-\gamma) s+\gamma^{d} \bar{\alpha}_{V} \circ \underline{\alpha}_{Y}^{-1} \circ\left(\frac{\mathbb{I}-\alpha_{Y} \circ \bar{\alpha}_{Y}^{-1}}{\gamma}\right)^{(d)}(s) & \text { when } \gamma \in(0,1) \text { and } d \in \mathbb{Z}_{>0} \\ \bar{\alpha}_{V} \circ \underline{\alpha}_{Y}^{-1} \circ\left(\mathbb{I}-\alpha_{Y} \circ \bar{\alpha}_{Y}^{-1}\right)^{(d)}(s) & \text { when } \gamma=1 \text { and } d \in \mathbb{Z}_{>0} \\ (1-\gamma) s & \text { when } \gamma \in(0,1) \text { and } d=\infty\end{cases} \end{aligned}$

Then, there exist $K, \lambda>0$, such that for any $\gamma \in\left(\gamma^{*}, 1\right]$, $d \in\left(d^{*}, \infty\right]$, for any $x \in \mathbb{R}^{n}$, the solution $\phi(\cdot, x)$ to system (5) satisfies $\sigma(\phi(k, x)) \leq K \sigma(x) e^{-\lambda k}$ for all $k \in \mathbb{Z}_{\geq 0}$.

Corollary 2 ensures a uniform global exponential stability property of $\{x: \sigma(x)=0\}$. It also provides explicit conditions on the pair $\left(\gamma^{*}, d^{*}\right)$ under which stability is guaranteed. Indeed, we either first fix $\gamma^{*} \in(\bar{\gamma}, 1]$ with $\bar{\gamma}=1-\frac{a_{W}}{\bar{a}_{V}+\bar{a}_{W}}$ and then select $d^{*}$ such that (15) holds, or we first fix $d^{*}>\bar{d}$ with $\bar{d}=\left\lfloor\frac{\ln \left(\bar{a}_{V}\left(\bar{a}_{V}+\bar{a}_{W}\right) / a_{W}^{2}\right)}{-\ln \left(1-\frac{a_{W}}{\bar{a}_{V}+\bar{a}_{W}}\right)}\right\rfloor$ and select $\gamma^{*}$ such that (15) holds. The resulting pair $\left(\gamma^{*}, d^{*}\right)$ is a suitable candidate for (15) by construction.

\section{Comparison of the conditions on $\gamma$ and $d$ with existing results}

It is difficult to compare the conditions on $\gamma$ and $d$ in the general case of Theorem 2 with those in [6] when $\gamma=1$ and those in [21] when $d=\infty$. For this reason, in the next lemma, we compare the bounds derived from Corollary 2 either when $\gamma=1$ or $d=\infty$ with those given in Corollary 3 in [6], which we denote ${ }^{2}$ as $d_{[6]}$, and in Corollary 2 in [21], which we denote as $\gamma_{[21]}$, respectively.

Lemma 2: Under the conditions of Corollary 2, the following holds.

(i) When $\bar{a}_{W}<a_{W}, \bar{\gamma}<\gamma_{[21]}:=\frac{\bar{a}_{V}}{\bar{a}_{V}+a_{W}}$ where $\bar{\gamma}=$ $1-\frac{a_{W}}{\bar{a}_{V}+\bar{a}_{W}}$ is the bound on $\gamma$ given by (15) when $d=\infty$.

(ii) $\bar{d}=-\ln d_{[6]} / \ln \left(1-\frac{a_{W}}{\bar{a}_{V}+\bar{a}_{W}}\right)$ where ${ }^{3} d_{[6]}:=\frac{\bar{a}_{V}\left(\bar{a}_{V}+\bar{a}_{W}\right)}{a_{W}^{2}}$ and $\bar{d}=-\frac{\ln \left(\frac{\bar{a}_{V}\left(\bar{a}_{V}+\bar{a}_{W}\right)}{a_{W}^{2}}\right)}{\ln \left(1-\frac{a_{W}}{\bar{a}_{V}+\bar{a}_{W}}\right)}$ is the bound on $d$ given by

(15) when $\gamma=1$.

Item (i) of Lemma 2 implies that the minimum discount factor $\bar{\gamma}$ given by Corollary 2 when $d=\infty$ is strictly smaller than the bound found in Corollary 2 from [21] when $\bar{\alpha}_{W}<\alpha_{W}$. Item (ii) of Lemma 2 provides a direct relationship between the estimate horizon $d_{[6]}$ of Corollary 3 from [6] and our minimum horizon estimate $\bar{d}$. It can therefore be used to infer which bound is tighter.

We refer to our preliminary work [5] for the illustration of Lemma 2. There, we use Lemma 2 to the examples found in $[6,21]$ and obtained from $36 \%$ to $63 \%$ improvement in the estimation on the lower bound on $d$ for [6] and a $6 \%$ improvement on the bound on $\gamma$ compared to [21].

\footnotetext{
${ }^{2}$ We make a change of variable $N-1=d$ to align our cost function $J_{\gamma, d}$ with the one in [6].

${ }^{3}$ There is a slight abuse of notation, since $\bar{d}$ and $d_{[6]}$ are supposed to be integers.
}

\section{RELATIONSHIPS BETWEEN THE OPTIMAL VALUE FUNCTIONS}

As explained in the introduction, often the goal is to minimize either a discounted infinite-horizon cost or an undiscounted finite-horizon cost, but the mentioned algorithms minimize a finite-horizon discounted cost instead. In this context, it is natural to ask what is the relationship between the cost we originally aim at minimizing, i.e. the infinite-horizon discounted one, and the one we actually minimize, i.e. the finite-horizon one. The next theorem provides a relationship between these two costs, as well as between the discounted finite-horizon cost and the undiscounted finite-horizon cost. These results are obtained by exploiting Assumption 1.

Theorem 3: Let $\gamma \in(0,1], d \in \mathbb{Z}_{>0}, x \in \mathbb{R}^{n}$ and suppose that Assumption 1 holds. Then

$$
\left\{\begin{array}{l}
V_{\gamma, d}(x) \leq V_{\gamma, \infty}(x) \leq V_{\gamma, d}(x)+\gamma^{d} v_{\gamma, d}(x) \\
V_{\gamma, d}(x) \leq V_{1, d}(x) \leq V_{\gamma, d}(x)+(1-\gamma) \sum_{k=1}^{d} v_{\gamma, k}(x),
\end{array}\right.
$$

where $v_{\gamma, k}(x):=\bar{\alpha}_{V} \circ \underline{\alpha}_{Y}^{-1} \circ\left(\frac{\mathbb{I}-\alpha_{Y} \circ \bar{\alpha}_{Y}^{-1}}{\gamma}\right)^{(k)} \circ \bar{\alpha}_{Y}(\sigma(x))$ for $k \in\{1, \ldots, d\}$ and $\alpha_{Y}, \underline{\alpha}_{Y}, \bar{\alpha}_{Y} \in \mathcal{K}_{\infty}$ come from Theorem 1, see Table I.

Theorem 3 provides explicit computable bounds on $V_{\gamma, \infty}$ (and $V_{1, d}$ ) based on $V_{\gamma, d}(x), \gamma, d$ and $\sigma(x)$, which can be used to evaluate the mismatch induced by the minimization of a finite-horizon discounted cost instead of an infinite-horizon discounted one (and an undiscounted finite-horizon one). The inequalities in (16) become equalities in the limit case, i.e. when $d \rightarrow \infty$ in (16a) and $\gamma \rightarrow 1$ in (16b). This is obviously true for (16b). The case of (16a) is addressed in the next lemma, which indeed ensures that $V_{\gamma, d}(x)$ tends to $V_{\gamma, \infty}(x)$ when $d \rightarrow \infty$, for $\gamma$ sufficiently close to 1 .

Lemma 3: Let $\Delta>0, x \in\left\{z \in \mathbb{R}^{n}: \sigma(z) \leq \Delta\right\}$. Suppose Assumption 1 holds and consider $\alpha_{Y}, \bar{\alpha}_{Y}, \underline{\alpha}_{Y}$ from Theorem 1. For $\gamma \in\left(1-\frac{\alpha_{Y}(\Delta)}{\bar{\alpha}_{Y}(\Delta)}, 1\right], V_{\gamma, d}(x)+\gamma^{d} v_{\gamma, d}(x) \rightarrow V_{\gamma, \infty}(x)$ when $d \rightarrow \infty$, where $v_{\gamma, d}(x)=\bar{\alpha}_{V} \circ \underline{\alpha}_{Y}^{-1} \circ\left(\frac{\mathbb{I}-\alpha_{Y} \circ \bar{\alpha}_{Y}^{-1}}{\gamma}\right)^{(d)} \circ$ $\bar{\alpha}_{Y}(\sigma(x))$.

When comparing (16a) with the corresponding error bounds usually found in approximate dynamic programming [1], we observe significant improvement when $\gamma$ is close to 1 . Indeed, in algorithms like value iteration, it is commonly assumed that $\ell(x, u)$ is bounded, e.g. $\ell(x, u) \in[0,1]$ for any $(x, u) \in \mathcal{W}$, which is not the case here. This property is used to derive that $V_{\gamma, \infty}(x) \leq V_{\gamma, d}(x)+\sum_{k=d}^{\infty} \gamma^{k} \leq V_{\gamma, d}(x)+\frac{\gamma^{d}}{1-\gamma}$. The term $\frac{\gamma^{d}}{1-\gamma}$, 
which serves as a near-optimality bound, clearly diverges to infinity when $\gamma \rightarrow 1$. This is not the case with the bound in (16a), which is small whenever $d$ is large, or when $\sigma(x)$ is small, even when $\gamma$ is close to 1 .

The work in [8] also provides relationship between the undiscounted infinite-horizon optimal cost and the finitehorizon one. As explained in the introduction, the results of this section rely on different assumptions, namely we do not rely on a relaxed dynamic programming property (see Proposition 2.2 and Assumption 4.2 in [8]), but on stabilizability and detectability properties. Furthermore, we address discounted costs contrary to [8].

Finally, simpler relationships between the optimal value functions can be obtained under the conditions of Corollary 2, as stated next.

Corollary 3: Let $d \in \mathbb{Z}_{>0}, x \in \mathbb{R}^{n}$ and suppose that the conditions of Corollary 2 are satisfied. The following hold.

(i) For any $\gamma \in(0,1]$,

$$
V_{\gamma, d}(x) \leq V_{\gamma, \infty}(x) \leq V_{\gamma, d}(x)+\hat{v}_{d}(x)
$$

where $\hat{v}_{d}(x):=\frac{\bar{a}_{V}\left(\bar{a}_{V}+\bar{a}_{W}\right)}{a_{W}}\left(1-\frac{a_{W}}{\bar{a}_{V}+\bar{a}_{W}}\right)^{d} \sigma(x)$.

(ii) For any $\gamma \in\left(1-\frac{a_{W}}{\bar{a}_{V}+\bar{a}_{W}}, 1\right]$,

$$
V_{\gamma, d}(x) \leq V_{1, d}(x) \leq V_{\gamma, d}(x)+S_{v}(x)
$$

where $S_{v}(x):=(1-\gamma) \frac{\bar{a}_{V}\left(\bar{a}_{V}+\bar{a}_{W}\right)^{2}}{a_{W}} \frac{\left(1-\frac{a_{W}}{a_{V}+\bar{a}_{W}}\right)}{a_{W}-(1-\gamma)\left(\bar{a}_{V}+\bar{a}_{W}\right)} \sigma(x)$.

The mismatch $\hat{v}_{d}(x)$ between $V_{\gamma, d}(x)$ and $V_{\gamma, \infty}(x)$ in (17) is linear in $\sigma(x)$ and no longer depends on $\gamma$ as in (16a). Furthermore, $\hat{v}_{d}(x) \rightarrow 0$ as $d \rightarrow \infty$ since $1-\frac{a_{W}}{\bar{a}_{V}+\bar{a}_{W}} \in(0,1)$ as shown in the proof of Corollary 1. Similarly, the mismatch $S_{v}(x)$ between $V_{\gamma, d}(x)$ and $V_{1, d}(x)$ is linear in $\sigma(x)$ and no longer depends on $d$ as in (16b). Moreover, $S_{v}(x) \rightarrow 0$ as $\gamma \rightarrow 1$ for any $x \in \mathbb{R}^{n}$.

\section{NeAR-Optimal SEQUENCE of InPUtS}

In this section, we explore whether an approximated solver for optimal cost (3) preserves our previous results in some sense. Hence, we study the case the available inputs sequence is only near-optimal for the discounted finite-horizon cost in the following sense.

Assumption 3: There exists a continuous function $\eta$ : $[0,+\infty) \times \mathcal{P} \rightarrow \mathbb{R}_{\geq 0}$, where $\mathcal{P}:=\left((0,1] \times \mathbb{Z}_{\geq 0}\right) \cup((0,1) \times$ $\left(\mathbb{Z}_{\geq 0} \cup\{+\infty\}\right)$ with $\eta(\cdot, \gamma, d) \in \mathcal{K}_{\infty}$ for any $(\gamma, d) \in \mathcal{P}$, such that $V_{\gamma, d}(x) \leq \widehat{V}_{\gamma, d}(x) \leq V_{\gamma, d}(x)+\eta(\epsilon, \gamma, d)$.

Assumption 3 means that, for any $x \in \mathbb{R}^{n}$ and $(\gamma, d) \in$ $\mathcal{P}$, we know a near-optimal sequence of admissible inputs $\hat{\boldsymbol{u}}_{\gamma, d}(x)$ where $\eta(\epsilon, \gamma, d)$ is the near-optimality bound. The constant error $\eta$ can be controlled by choosing parameter $\epsilon>0$ depending on $(\gamma, d)$ as $\eta(\cdot, \gamma, d)$ is of class $\mathcal{K}_{\infty}$. Assumption 3 covers near-optimality bounds of the form $\eta(\epsilon, \gamma, d)=\frac{1-\gamma^{d}}{1-\gamma} \epsilon$ where $\epsilon$ is related to approximation errors; such bounds are commonly found in the approximate dynamic programming literature, see [1]. The function $\eta$ in Assumption 3 takes value in $\mathbb{R}_{\geq 0} \times \mathcal{P}$, and not in $\mathbb{R}_{\geq 0} \times[0,1] \times\left(\mathbb{Z}_{\geq 0} \cup\{+\infty\}\right)$ as we might expect, to cover this type of bounds, which explodes when $(\gamma, d) \rightarrow(1, \infty)$. Compared to [10], where stability is analysed for control inputs generated by approximate value iteration, we: (i) study stability of a generic closed set, and not only of the origin; (ii) do not need the explicit knowledge of a globally stabilizing policy; (iii) address discounted costs; (iv) the stage cost is not necessarily quadratic; (v) allow for a constant error $\eta(\epsilon, \gamma, d)$, which therefore does not depend on $x$.

We write system (1) in closed-loop with a near-optimal sequence of inputs as

$$
x_{k+1} \in f\left(x_{k}, \widehat{\mathcal{U}}_{\gamma, d}\left(x_{k}\right)\right)=: \widehat{F}_{\gamma, d}\left(x_{k}\right),
$$

and we denote by $\hat{\phi}(k, x)$ a solution to (19) at time $k \in \mathbb{Z}_{\geq 0}$ with initial condition $x \in \mathbb{R}^{n}$.

The next theorem provides conditions under which the stability of system (5) follows.

Theorem 4: Consider system (19) and suppose Assumptions 1 and 3 hold. There exists $\beta \in \mathcal{K} \mathcal{L}$ such that for any $\delta, \Delta>0$, there exist $\left(\gamma^{*}, d^{*}\right) \in(0,1) \times \mathbb{Z}_{\geq 0}$ such that for any $(\gamma, d) \in$ $\mathcal{P}_{\gamma^{*}, d^{*}}:=\left(\left(\gamma^{*}, 1\right] \times\left(d^{*}, \infty\right]\right) \cap \mathcal{P}$, there exists $\epsilon^{*}>0$, such that for any $\epsilon \in\left[0, \epsilon^{*}\right)$ and $x \in\left\{z \in \mathbb{R}^{n}: \sigma(z) \leq \Delta\right\}$, any solution $\hat{\phi}(\cdot, x)$ to system (19) satisfies

$$
\sigma(\hat{\phi}(k, x)) \leq \max \{\beta(\sigma(x), k), \delta\} \quad \forall k \in \mathbb{Z}_{\geq 0}
$$

Moreover, when $\eta(\epsilon, \gamma, d)$ is non-increasing in $\gamma$ and $d$, given $\delta, \Delta>0$, there exists $\left(\epsilon^{*}, \gamma^{*}, d^{*}\right) \in \mathbb{R}_{>0} \times(0,1) \times \mathbb{Z}_{\geq 0}$ such that for any $(\epsilon, \gamma, d) \in\left[0, \epsilon^{*}\right) \times \mathcal{P}_{\gamma^{*}, d^{*}},(20)$ holds.

Theorem 4 ensures a semiglobal practical stability property where the adjustable parameters are not only $\gamma, d$ as before, but also $\epsilon$. We stress that $\epsilon^{*}$, the upper-bound on $\epsilon$, has to be chosen as a function of $(\gamma, d)$ in general, not $\left(\gamma^{*}, d^{*}\right)$. This is due to the fact that the error term $\eta(\epsilon, \gamma, d)$ is potentially increasing and unbounded in $\gamma$ and $d$. This is the case for instance when $\eta(\epsilon, \gamma, d)=\frac{1-\gamma^{d}}{1-\gamma} \epsilon$, which explodes as $(\gamma, d) \rightarrow(1, \infty)$ as already mentioned. We are thus forced to adapt $\epsilon$ to $(\gamma, d)$. This is no longer the case when $\eta(\epsilon, \gamma, d)$ is non-increasing in $\gamma$ and $d$, as stated in the last part of Theorem 4 .

While the determination of the pair $\left(\gamma^{*}, d^{*}\right)$ such that (20) holds is as difficult as in Theorem 2, the next lemma states that any pair $\left(\gamma^{*}, d^{*}\right)$ such that (20) holds for given $(\delta, \Delta)$, ensures that (11) holds with tuple $\left(\gamma^{*}, d^{*}, \delta, \Delta\right)$, but the reverse may not be true.

Lemma 4: Let $\left(\gamma^{*}, d^{*}, \delta, \Delta\right)$ be such that Theorem 4 holds for some $\eta$ and $\epsilon^{*}$. Then Theorem 2 is verified with the same tuple $\left(\gamma^{*}, d^{*}, \delta, \Delta\right)$.

Lemma 4 is useful in practice to have lower bounds on $\gamma^{*}$ and $d^{*}$ when the considered algorithm produces near-optimal inputs according to Assumption 3, as illustrated in the next section.

Regarding optimality, the following corollary provides a relationship between the infinite-horizon discounted optimal cost $V_{\gamma, \infty}(x)$ and $\widehat{V}_{\gamma, d}(x)$.

Corollary 4: Suppose that Assumption 1 and 3 hold, let $\epsilon \geq 0,(\gamma, d) \in \mathcal{P}$ and $x \in \mathbb{R}^{n}$ and consider $v_{\gamma, d}(x)$ defined in Theorem 3, it holds that

$$
\widehat{V}_{\gamma, d}(x)-\eta(\epsilon, \gamma, d) \leq V_{\gamma, \infty}(x) \leq \widehat{V}_{\gamma, d}(x)+\gamma^{d} v_{\gamma, d}(x)
$$




\section{ILLUSTRATIVE EXAMPLE}

We consider the model of an inverted pendulum discretized by an Euler scheme with sampling period $T>0$,

$$
\begin{aligned}
& x_{1}^{+}=x_{1}+T x_{2} \\
& x_{2}^{+}=x_{2}+T\left(a \sin \left(x_{1}\right)-b x_{2}+c u\right)
\end{aligned}
$$

where $x_{1} \in \mathbb{R}$ is the angular position of the pendulum, with $x_{1}=0$ being the upper-position, $x_{2} \in \mathbb{R}$ the angular velocity and $u \in \mathbb{R}$ is a controllable torque at the rotation axis. The constants $a, b, c>0$ are related to the mass, the dissipation and the motor gain, respectively. We have $\left(x_{1}, x_{2}\right)=x \in \mathbb{R}^{2}$ and $u \in \mathcal{U}(x)=\mathbb{R}$. Let $\sigma(x)=\left|x_{1}\right|+\left|x_{2}\right|$ and consider cost (2) with $\ell(x, u)=\sigma(x)+r|u|$ for some $r>0$, for any $(x, u) \in \mathbb{R}^{2} \times \mathbb{R}$. First, we verify that SA holds by applying Theorem 1 and item $d_{3}$ ) of Theorem 2 in [14]. We have that items a) to c) of Theorem 1 in [14] hold. Item $d_{3}$ ) of Theorem 2 in [14] is satisfied since $|u| \leq \ell(x, u)$, thus $|u| \rightarrow \infty, \ell(x, u) \rightarrow \infty$ for any $x \in \mathbb{R}^{2}$. For item e) of Theorem 1 in [14], let $x \in \mathbb{R}^{2}$ and consider the infinite sequence $v(x):=\left[\frac{1}{c}\left(-a \sin \left(x_{1}\right)+\right.\right.$ $\left.\left.b x_{2}-\frac{x_{1}+T x_{2}}{T^{2}}\right), \frac{1}{c}\left(-a \sin \left(x_{1}+T x_{2}\right)+b \frac{x_{1}+T x_{2}}{T}\right), 0, \ldots\right]$. It follows that $\phi\left(1, x,\left.v(x)\right|_{1}\right)=\left(x_{1}+T x_{2},-\frac{x_{1}+T x_{2}}{T}\right)$, and $\phi\left(k, x,\left.v(x)\right|_{k}\right)=0$ for $k \geq 2$. For any $\gamma \in(0,1]$, we have

$$
\begin{aligned}
& J_{\gamma, \infty}(x, v(x)) \\
& =\left|x_{1}\right|+\left|x_{2}\right|+r\left|\frac{1}{c}\left(-a \sin \left(x_{1}\right)+b x_{2}-\frac{x_{1}+T x_{2}}{T^{2}}\right)\right| \\
& +\gamma\left(\left|x_{1}+T x_{2}\right|+\left|\frac{x_{1}}{T}+x_{2}\right|\right. \\
& \left.+r\left|\frac{1}{c}\left(-a \sin \left(x_{1}+T x_{2}\right)+b \frac{x_{1}+T x_{2}}{T}\right)\right|\right) \\
& \leq\left|x_{1}\right|+\left|x_{2}\right|+\frac{r}{c}\left(a\left|x_{1}\right|+b\left|x_{2}\right|+\frac{1}{T^{2}}\left|x_{1}\right|+\frac{1}{T}\left|x_{2}\right|\right) \\
& +\gamma\left(\left|x_{1}\right|+T\left|x_{2}\right|+\frac{1}{T}\left|x_{1}\right|+\left|x_{2}\right|\right. \\
& +\frac{r}{c}\left(a\left(\left|x_{1}\right|+T\left|x_{2}\right|\right)+b\left(\frac{1}{T}\left|x_{1}\right|+\left|x_{2}\right|\right)\right) \\
& \leq \theta_{1}(\gamma, r, T)\left|x_{1}\right|+\theta_{2}(\gamma, r, T)\left|x_{2}\right|,
\end{aligned}
$$

with $\theta_{1}(\gamma, r, T):=1+\frac{r a}{c}+\frac{r}{c T^{2}}+\gamma\left(1+\frac{1}{T}+\frac{r a}{c}+\frac{r b}{c T}\right)$ and $\theta_{2}(\gamma, r, T):=1+\frac{r b}{c}+\frac{r}{c T}+\gamma\left(T+1+\frac{r T}{c}+\frac{r b}{c}\right)$, which is finite for all $T>0$. Thus, Theorem 2 of [14] is verified and SA holds. Furthermore, since $J_{1, \infty}(x, v(x)) \leq \theta_{1}(1, r, T)\left|x_{1}\right|+$ $\theta_{2}(1, r, T)\left|x_{2}\right|$ we verify item (i) of Assumption 1 is satisfied with $\bar{\alpha}_{V}=\max \left\{\theta_{1}(1, r, T), \theta_{2}(1, r, T)\right\} \mathbb{I}$. From $\ell(x, u) \geq$ $\ell(x, 0)=\sigma(x)$, item (ii) of Assumption 1 is verified with $W=\bar{\alpha}_{W}=0$ and $\alpha_{W}=\mathbb{I}$.

In the following, we take $a=b=c=1$, with time-step $T=1$ and $r=1$. In this case, $\bar{\alpha}_{V}=7 \mathbb{I}$. In view of Corollary 2, we find $\bar{d}=\left\lfloor\frac{0-\ln 7^{2}}{\ln 6-\ln 7}\right\rfloor=25$ and $\bar{\gamma}=\frac{6}{7}$, thus global exponential stability is guaranteed for any pair $\left(\gamma^{*}, d^{*}\right)$ with $\gamma^{*} \geq \bar{\gamma}$ and $d^{*} \geq \bar{d}$ when the optimal sequence of inputs is applied to system (22). This analysis is of course conservative, and a different derivation of $\bar{\alpha}_{V}$ might provide different bounds on $\left(\gamma^{*}, d^{*}\right)$.

Because we do not know how to compute optimal sequences of inputs, we use a scheme based on approximate value iteration to generate the inputs. This scheme relies on a simple finite difference approximation, with $N=33^{2}$ points equally distributed in $[-\pi, \pi] \times[-\pi, \pi]$ for the state space, and 101 equally distributed quantized inputs in $[-10,10]$ centered at 0 . As a result, Assumption 3 holds for some $\eta$, which can be derived by adapting the results from [2] for aggregation ${ }^{4}$ per Theorem 4, system (22) controlled by such scheme satisfies (20). Figure 1 provides plots of $\sigma(\hat{\phi}(\cdot, x))$ for the initial condition $(3,0)$ for different values of $(\gamma, d)$. As expected, when $\gamma$ or $d$ are too small, $\sigma(\hat{\phi}(\cdot, x))$ does not converge to a "small" neighborhood of the origin, where we recall that $\hat{\phi}(\cdot, x)$ is the solution of (19) controlled by the near-optimal sequence. To analyse the impact of $(\gamma, d)$ on the closed-loop system, we consider three initial conditions, namely $(\pi, 0),\left(\frac{\pi}{2}, \frac{\pi}{2}\right)$ and $\left(\frac{\pi}{10}, 0.1\right)$, for different pairs $(\gamma, d)$ and we have checked numerically whether the state measure converges to the set $\mathcal{S}:=\left\{z \in \mathbb{R}^{2}: \sigma(z) \leq 0.5\right\}$ in 11 steps, and that it stays in $\mathcal{S}$ for at least 40 steps. The obtained results are summarized in Fig. 2. As expected by Theorem 4, we see in Fig. 2 that both $(\gamma, d)$ have to be sufficiently large for $\sigma(\hat{\phi}(\cdot, x))$ to converge close to the origin. Furthermore, Theorem 4 suggests that stability may be lost if $\epsilon$ is not small enough for given $(\gamma, d)$, this is not apparent for our test case with $N=33^{2}$. However, by reducing the number of points for the state space from $N=33^{2}$ to $N=31^{2}$, we observe a degradation of our convergence test for $\gamma$ to close to 1 and $d$ too large, see Fig. 3. This further illustrates the statement made in Theorem 4 in extension to those made in Theorem 2. Namely, that pair $(\gamma, d)$ cannot be simply taken close to $(1, \infty)$, as they have a role in addition to $\epsilon$ in keeping the error term $\eta$ in check.

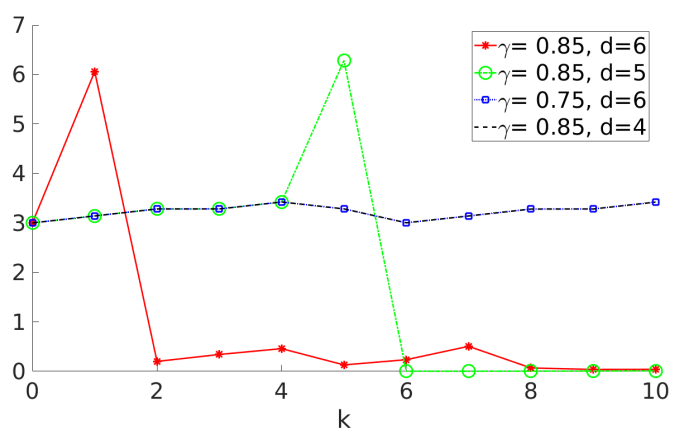

Figure 1: $\sigma(\hat{\phi}(\cdot, x))$ for 4 different pairs $(\gamma, d)$ and $x=(3,0)$.

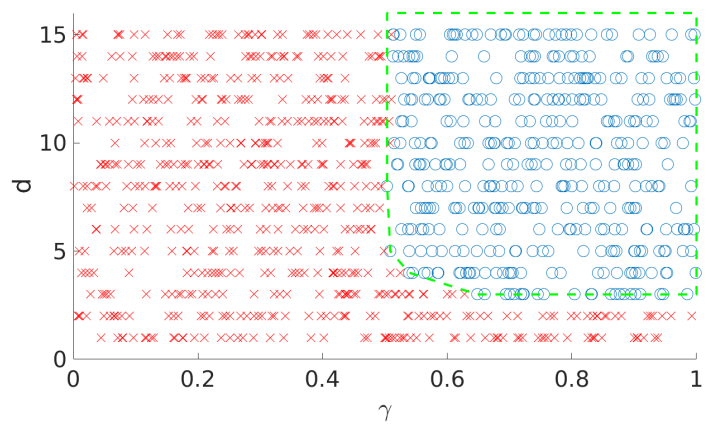

Figure 2: Convergence testing of 1000 sample pairs $(\gamma, d)$, $N=33^{2}$. Symbol o denotes convergence to $\mathcal{S}$, while $\times$ the converse.

${ }^{4}$ The detailed derivations are not provided for space reasons. 


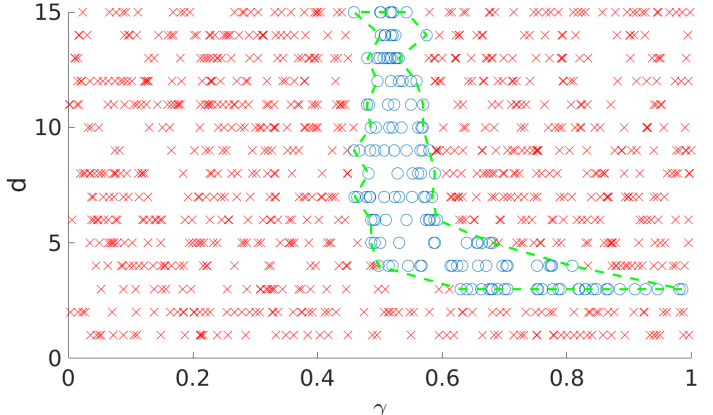

Figure 3: Convergence testing of 1000 sample pairs $(\gamma, d)$, $N=31^{2}$. Symbol $\circ$ denotes convergence to $\mathcal{S}$, while $\times$ the converse.

\section{PROOFS}

\section{A. Proof of Theorem 1} $d$.

We distinguish three cases depending on the value of $\gamma$ and

Case 1: $\gamma \in(0,1)$ and $d \in \mathbb{Z}_{>0}$.

Let $\gamma \in(0,1), d \in \mathbb{Z}_{>0}, x \in \mathbb{R}^{n}$ and $v \in F_{\gamma, d}^{*}(x)$. There exists $\left[u_{0}^{*}, u_{1}^{*}, \ldots, u_{d}^{*}\right]=\boldsymbol{u}_{\gamma, d}^{*}(x)$ such that $v=f\left(x, u_{0}^{*}\right)$ and $\boldsymbol{u}_{\gamma, d}^{*}(x)$ is an optimal input sequence for system (1) with cost (2). Hence $V_{\gamma, d}(x)=J_{\gamma, d}\left(x, \boldsymbol{u}_{\gamma, d}^{*}(x)\right)$.

The proof can be summarized as follows. We start by showing item (ii) of Theorem 1. For this, we first upper-bound $V_{\gamma, d}(v)$ using Bellman equation. We then derive a preliminary upper-bound on $V_{\gamma, d}(v)-V_{\gamma, d}(x)$. Afterwards, We define $Y_{\gamma, d}:=V_{\gamma, d}+W$ where $W$ comes from Assumption 1 and we derive the desired result.

Since stage cost $\ell$ is nonnegative and in view of item (i) of Assumption 1,

$$
\ell\left(x, u_{0}^{*}\right) \leq V_{\gamma, d}(x) \leq \bar{\alpha}_{V}(\sigma(x)) .
$$

Let $j \in\{1, \ldots, d\}$. Consider the sequence $\hat{\boldsymbol{u}} \quad:=\left[u_{1}^{*}, u_{2}^{*}, \ldots, u_{d-j}^{*}, \overline{\boldsymbol{u}}_{j}\right]$ where $\overline{\boldsymbol{u}}_{j}:=$ $\boldsymbol{u}_{\gamma, j}^{*}\left(\phi\left(d-j+1, x,\left.\boldsymbol{u}_{\gamma, d}^{*}(x)\right|_{d-j+1}\right)\right),\left.\quad \boldsymbol{u}_{\gamma, d}^{*}(x)\right|_{d-j+1}=$ $\left[u_{0}^{*}, \ldots, u_{d-j}^{*}\right]$ and $\phi$ denotes the solution of system (1). The sequence $\hat{\boldsymbol{u}}$ consists of the first $d-j$ elements of $\boldsymbol{u}_{\gamma, d}^{*}(x)$ after $u_{0}^{*}$, followed by an optimal input sequence of length $j+1$ for cost $J_{\gamma, d-j}$ at state $\phi\left(d-j+1, x,\left.\boldsymbol{u}_{\gamma, d}^{*}(x)\right|_{d-j+1}\right)$. Note that the sequence $\overline{\boldsymbol{u}}_{j}$ exist and minimizes $J_{\gamma, j}\left(\phi\left(d-j+1, x,\left.\boldsymbol{u}_{\gamma, d}^{*}(x)\right|_{d-j+1}\right), \overline{\boldsymbol{u}}_{j}\right)$ from SA. From the definition of cost $J_{\gamma, d}$ in (2) and $V_{\gamma, d}$ in (3), $V_{\gamma, d}(v) \leq J_{\gamma, d}(v, \hat{\boldsymbol{u}})=J_{\gamma, d-j-1}\left(v,\left.\hat{\boldsymbol{u}}\right|_{d-j}\right)+$ $\gamma^{d-j} J_{\gamma, j}\left(\phi\left(d-j, v,\left.\hat{\boldsymbol{u}}\right|_{d-j}\right), \overline{\boldsymbol{u}}_{j}\right)$. By definition of $\overline{\boldsymbol{u}}_{j}$, $V_{\gamma, d}(v) \leq \sum_{k=0}^{d-j-1} \gamma^{k} \ell\left(\phi\left(k, v,\left.\hat{\boldsymbol{u}}\right|_{k}\right), \hat{\boldsymbol{u}}_{k}\right)+\gamma^{d-j} V_{\gamma, j}(\phi(d-$ $\left.\left.j, v,\left.\hat{\boldsymbol{u}}\right|_{d-j}\right)\right)$. For any $k \in\{0, \ldots, d\}, \phi\left(k, v,\left.\hat{\boldsymbol{u}}\right|_{k}\right)=$ $\phi\left(k+1, x,\left.\left[u_{0}^{*}, \hat{\boldsymbol{u}}\right]\right|_{k+1}\right)=\phi\left(k+1, x,\left.\boldsymbol{u}_{\gamma, d}^{*}(x)\right|_{k+1}\right)$. Similarly, since $j \in\{1, \ldots, d\}$ implies $0 \leq d-j<d$, $\phi\left(d-j, v,\left.\hat{\boldsymbol{u}}\right|_{d-j}\right)=\phi\left(d-j+1, x,\left.\boldsymbol{u}_{\gamma, d}^{*}(x)\right|_{d-j+1}\right)$. Thus

$$
\begin{aligned}
V_{\gamma, d}(v) \leq & \sum_{k=0}^{d-j-1} \gamma^{k} \ell\left(\phi\left(k+1, x,\left.\boldsymbol{u}_{\gamma, d}^{*}\right|_{k+1}\right),\left[\boldsymbol{u}_{\gamma, d}^{*}(x)\right]_{k+1}\right) \\
& +\gamma^{d-j} V_{\gamma, j}\left(\phi\left(d-j+1, x,\left.\boldsymbol{u}_{\gamma, d}^{*}(x)\right|_{d-j+1}\right)\right),
\end{aligned}
$$

where $\left[\boldsymbol{u}_{\gamma, d}^{*}(x)\right]_{k+1}=u_{k+1}^{*}$. Using the following shorthand notation, we define the optimal solution $\phi_{k}^{*}:=\phi\left(k, x, \boldsymbol{u}_{\gamma, d}^{*}(x)\right)$ and the optimal stage cost $\ell_{k}^{*}:=\ell\left(\phi_{k}^{*}, u_{k}^{*}\right)$ for $k \in\{0, \ldots, d\}$. Hence,

$$
V_{\gamma, d}(v)=V_{\gamma, d}\left(\phi_{1}^{*}\right) \leq \sum_{k=0}^{d-j-1} \gamma^{k} \ell_{k+1}^{*}+\gamma^{d-j} V_{\gamma, j}\left(\phi_{d-j+1}^{*}\right) .
$$

Furthermore from the definition of $V_{\gamma, d}(x)$,

$$
V_{\gamma, d}(x)=\sum_{k=0}^{d} \gamma^{k} \ell_{k}^{*}
$$

and

$$
V_{\gamma, d}(x)-\ell_{0}^{*} \geq \gamma \sum_{k=0}^{d-j-1} \gamma^{k} \ell_{k+1}^{*}
$$

Subtracting (26) from (25), it follows $V_{\gamma, d}(v)-V_{\gamma, d}(x) \leq$ $-\sum_{k=0}^{d} \gamma^{k} \ell_{k}^{*}+\sum_{k=0}^{d-j-1} \gamma^{k} \ell_{k+1}^{*}+\gamma^{d-j} V_{\gamma, j}\left(\phi_{d-j+1}^{*}\right)=$ $-\ell_{0}^{*}+(1-\gamma) \sum_{k=0}^{d-j-1} \gamma^{k} \ell_{k+1}^{*}+\gamma^{d-j} V_{\gamma, j}\left(\phi_{d-j+1}^{*}\right)$. In view of (27), we have $\sum_{k=0}^{d-j-1} \gamma^{k} \ell_{k+1}^{*} \leq \frac{V_{\gamma, d}(x)-\ell_{0}^{*}}{\gamma}$, hence

$$
\begin{aligned}
& V_{\gamma, d}(v)-V_{\gamma, d}(x) \\
& \quad \leq-\ell_{0}^{*}-\frac{1-\gamma}{\gamma} \ell_{0}^{*}+\frac{1-\gamma}{\gamma} V_{\gamma, d}(x)+\gamma^{d-j} V_{\gamma, j}\left(\phi_{d-j+1}^{*}\right) \\
& =-\frac{\ell_{0}^{*}}{\gamma}+\frac{1-\gamma}{\gamma} V_{\gamma, d}(x)+\gamma^{d-j} V_{\gamma, j}\left(\phi_{d-j+1}^{*}\right) .
\end{aligned}
$$

From item (i) of Assumption 1,

$$
\begin{aligned}
& V_{\gamma, d}(v)-V_{\gamma, d}(x) \\
& \quad \leq-\frac{\ell_{0}^{*}}{\gamma}+\frac{1-\gamma}{\gamma} V_{\gamma, d}(x)+\gamma^{d-j} \bar{\alpha}_{V}\left(\sigma\left(\phi_{d-j+1}^{*}\right)\right) \\
& \quad=\frac{1}{\gamma}\left(-\ell_{0}^{*}+(1-\gamma) V_{\gamma, d}(x)+\gamma^{d-j+1} \bar{\alpha}_{V}\left(\sigma\left(\phi_{d-j+1}^{*}\right)\right)\right) .
\end{aligned}
$$

Adding and subtracting $\frac{1-\gamma}{\gamma} W(x)$,

$$
\begin{aligned}
& V_{\gamma, d}(v)-V_{\gamma, d}(x) \\
& \leq \leq \frac{1}{\gamma}\left(-\ell_{0}^{*}-(1-\gamma) W(x)+(1-\gamma)\left(V_{\gamma, d}(x)+W(x)\right)\right. \\
& \left.\quad+\gamma^{d-j+1} \bar{\alpha}_{V}\left(\sigma\left(\phi_{d-j+1}^{*}\right)\right)\right) .
\end{aligned}
$$

Let $Y_{\gamma, d}=V_{\gamma, d}+W$. In view of item (ii) of Assumption 1 and since $\gamma \leq 1, \gamma W(v)-W(x) \leq-\alpha_{W}(\sigma(x))+\ell_{0}^{*}$. Dividing everything by $\gamma$ and since $\frac{1}{\gamma}=1+\frac{1-\gamma}{\gamma}, W(v)-W(x)-$ $\frac{1-\gamma}{\gamma} W(x) \leq-\frac{\alpha_{W}(\sigma(x))}{\gamma}+\frac{\ell_{0}^{\gamma}}{\gamma}$. Therefore,

$$
W(v)-W(x) \leq \frac{1}{\gamma}\left(-\alpha_{W}(\sigma(x))+\ell_{0}^{*}+(1-\gamma) W(x)\right) .
$$

In view of (29) and (30),

$$
\begin{aligned}
& Y_{\gamma, d}(v)-Y_{\gamma, d}(x) \\
& \leq \frac{1}{\gamma}\left(-\ell_{0}^{*}-(1-\gamma) W(x)+(1-\gamma) Y_{\gamma, d}(x)\right. \\
& \left.\quad+\gamma^{k^{*}} \bar{\alpha}_{V}\left(\sigma\left(\phi_{k^{*}}^{*}\right)\right)-\alpha_{W}(\sigma(x))+\ell_{0}^{*}+(1-\gamma) W(x)\right) \\
& =\frac{1}{\gamma}\left(-\alpha_{W}(\sigma(x))+(1-\gamma) Y_{\gamma, d}(x)+\gamma^{k^{*}} \bar{\alpha}_{V}\left(\sigma\left(\phi_{k^{*}}^{*}\right)\right)\right)
\end{aligned}
$$


where $k^{*}:=d-j+1$. A major difficulty compared to [6] is how to bound $\sigma\left(\phi_{k^{*}}^{*}\right)$ in (31) because of the discount factor. For this purpose, we use Theorem 5 given in the Appendix. From item (ii) of Theorem 5 , it follows that, for any $k \in\{0, \ldots, d-1\}$,

$$
\begin{aligned}
& Y_{\gamma, d-(k+1)}\left(\phi_{k+1}^{*}\right)-Y_{\gamma, d-k}\left(\phi_{k}^{*}\right) \\
& \quad \leq \frac{1}{\gamma}\left(-\alpha_{Y}\left(\sigma\left(\phi_{k}^{*}\right)\right)+(1-\gamma) Y_{\gamma, d-k}\left(\phi_{k}^{*}\right)\right) .
\end{aligned}
$$

We write

$$
Y_{\gamma, d-(k+1)}\left(\phi_{k+1}^{*}\right) \leq \frac{-\alpha_{Y} \circ \bar{\alpha}_{Y}^{-1}\left(Y_{\gamma, d-k}\left(\phi_{k}^{*}\right)\right)+Y_{\gamma, d-k}\left(\phi_{k}^{*}\right)}{\gamma}
$$

where $\bar{\alpha}_{Y}=\bar{\alpha}_{V}+\bar{\alpha}_{W}$ and $\alpha_{Y}=\alpha_{W}$, since $\alpha_{Y}\left(\sigma\left(\phi_{k}^{*}\right)\right) \geq$ $\alpha_{Y} \circ \bar{\alpha}_{Y}^{-1}\left(Y_{\gamma, d-k}\left(\phi_{k}^{*}\right)\right)$, which follows from item (i) of Theorem 5. As explained in Remark 2, we can assume $\mathbb{I}-\alpha_{Y} \circ \bar{\alpha}_{Y}^{-1} \in \mathcal{K}_{\infty}$ without loss of generality. Thus, starting from $Y_{\gamma, d}(x)$ and proceeding by iteration, we have $Y_{\gamma, d-k^{*}}\left(\phi_{k^{*}}^{*}\right) \leq\left(\frac{\mathbb{I}-\alpha_{Y} \circ \bar{\alpha}_{Y}^{-1}}{\gamma}\right)^{\left(k^{*}\right)}\left(Y_{\gamma, d}(x)\right)$. We apply item (i) of Theorem 5 and conclude that

$$
\sigma\left(\phi_{k^{*}}^{*}\right) \leq \underline{\alpha}_{Y}^{-1}\left(\left(\frac{\mathbb{I}-\alpha_{Y} \circ \bar{\alpha}_{Y}^{-1}}{\gamma}\right)^{\left(k^{*}\right)}\left(Y_{\gamma, d}(x)\right)\right) .
$$

It follows from (31) and (33) that

$$
\begin{aligned}
& Y_{\gamma, d}(v)-Y_{\gamma, d}(x) \\
& \leq \frac{1}{\gamma}\left[-\alpha_{W}(\sigma(x))+(1-\gamma) Y_{\gamma, d}(x)\right. \\
&\left.+\gamma^{k^{*}} \bar{\alpha}_{V} \circ \underline{\alpha}_{Y}^{-1}\left(\left(\frac{\mathbb{I}-\alpha_{Y} \circ \bar{\alpha}_{Y}^{-1}}{\gamma}\right)^{\left(k^{*}\right)}\left(Y_{\gamma, d}(x)\right)\right)\right] .
\end{aligned}
$$

Thus, equation (10) is verified with $\alpha_{Y}=\alpha_{W} \in \mathcal{K}_{\infty}$ and $\Upsilon\left(s, \gamma, k^{*}\right)=(1-\gamma) s+\gamma^{k^{*}} \bar{\alpha}_{V} \circ \underline{\alpha}_{Y}^{-1}\left(\left(\frac{\mathbb{I}-\alpha_{Y} \circ \bar{\alpha}_{Y}^{-1}}{\gamma}\right){ }^{\left(k^{*}\right)}(s)\right)$ Recall that $j$ is freely selected in $\{1, \ldots, d\}$, as a result so is $k^{*} \in\{1, \ldots, d\}$. Note that $\Upsilon(s, \gamma, d) \geq 0$ for any $s \geq 0$, as $0 \leq \mathbb{I}-\alpha_{Y} \circ \bar{\alpha}_{Y}^{-1}$, which follows from $\alpha_{Y}=\underline{\alpha}_{Y} \leq \bar{\alpha}_{Y}$. Let $s \geq 0$, consider $(1-\gamma) s+\bar{\alpha}_{V}\left(\underline{\alpha}_{Y}^{-1}\left(\left(\frac{\mathbb{I}-\alpha_{Y} \circ \bar{\alpha}_{Y}^{-1}}{\gamma}\right)^{(d)}(s)\right)\right) \geq$ $\Upsilon(s, \gamma, d)$. Note that $s-\alpha_{Y} \circ \bar{\alpha}_{Y}^{-1}(s)<s$ if $s \neq 0$. Indeed, suppose $s-\alpha_{Y} \circ \bar{\alpha}_{Y}^{-1}(s)=s$ and $s \neq 0$, this is only possible if $\alpha_{Y} \circ \bar{\alpha}_{Y}^{-1}(s)=0$, we attain a contradiction. Hence, $s-$ $\alpha_{Y} \circ \bar{\alpha}_{Y}^{-1}(s)<s$ when $s>0$, and zero at zero. Therefore $(1-\gamma) s+\bar{\alpha}_{V}\left(\underline{\alpha}_{Y}^{-1}\left(\left(\frac{\mathbb{I}-\alpha_{Y} \circ \bar{\alpha}_{Y}^{-1}}{\gamma}\right)^{(d)}(s)\right)\right) \rightarrow 0$ when $\gamma \rightarrow 1$ and $d \rightarrow \infty$. Finally, recall that $0 \leq \Upsilon(s, \gamma, d)$. It follows, by the sandwich rule, that $\Upsilon(s, \gamma, d) \rightarrow 0$ when $\gamma \rightarrow 1$ and $d \rightarrow \infty$. Hence, item (ii) of Theorem 1 holds.

In view of Assumption 1, $Y_{\gamma, d} \leq \bar{\alpha}_{Y}(\sigma(x))$ with $\bar{\alpha}_{Y}=\bar{\alpha}_{V}+\bar{\alpha}_{W} \in \mathcal{K}_{\infty}$. From item (ii) of Assumption 1, we have $W(x) \geq \alpha_{W}(\sigma(x))-\ell\left(x, u_{0}^{*}\right)$. Associated with (23), it follows $Y_{\gamma, d} \geq \alpha_{W}(\sigma(x))-\ell\left(x, u_{0}^{*}\right)+\ell\left(x, u_{0}^{*}\right)=\alpha_{W}(\sigma(x))$. Thus $\underline{\alpha}_{Y}=\alpha_{W} \in \mathcal{K}_{\infty}$. Item (i) of Theorem 1 is satisfied.

Case 2: $\gamma=1$ and $d \in \mathbb{Z}_{>0}$

By following the steps of Case 1 with $\gamma=1$, the desired result is obtained.
Case 3: $\gamma \in(0,1)$ and $d=\infty$

Let $\gamma \in(0,1), x \in \mathbb{R}^{n}$ and $v \in F_{\gamma, \infty}^{*}(x)$. From Bellman equation, $V_{\gamma, \infty}(x)=\ell_{0}^{*}+\gamma V_{\gamma, \infty}(v)$, thus $V_{\gamma, \infty}(v)=\frac{-\ell_{0}^{*}+V_{\gamma, \infty}(x)}{\gamma}$. By following the steps of Case 1, the desired result is obtained.

\section{B. Proof of Theorem 2}

Let $\Delta, \delta>0, d \in\left(d^{*}, \infty\right]$ and $\gamma \in\left(\gamma^{*}, 1\right]$, where $\gamma^{*}, d^{*}$ are defined in the following, $x \in \mathbb{R}^{n}$ be such that $\sigma(x) \leq \Delta$, $v \in F_{\gamma, d}^{*}(x)$. There exists $\boldsymbol{u}_{\gamma, d}^{*}(x)$ with first element $u_{0}^{*}$ such that $v=f\left(x, u_{0}\right)$ according to SA.

Define $\widetilde{\Delta}:=\bar{\alpha}_{Y}(\Delta), \widetilde{\delta}:=\left(\mathbb{I}-\frac{\widetilde{\alpha}_{Y}}{2}\right)^{-1} \circ \underline{\alpha}_{Y}(\delta), \widetilde{\alpha}_{Y}:=$ $\alpha_{Y} \circ \bar{\alpha}_{Y}{ }^{-1}$, where $\alpha_{Y}, \bar{\alpha}_{Y}, \underline{\alpha}_{Y}$ come from Theorem 1. Let $\left(\gamma^{*}, d^{*}\right)$ such that the following holds for all $d^{\prime}>d^{*}, \gamma^{\prime} \in$ $\left(\gamma^{*} 1\right]$

$$
\forall s \in[\widetilde{\delta}, \widetilde{\Delta}], \quad \Upsilon\left(s, \gamma^{\prime}, d^{\prime}\right) \leq\left(1-\frac{\gamma^{\prime}}{2}\right) \widetilde{\alpha}_{Y}(s),
$$

where $\Upsilon$ comes from Theorem 1 . Such a pair $\left(\gamma^{*}, d^{*}\right)$ always exists for the following reason. Consider the function $\psi$ : $(\gamma, d) \mapsto \max _{s \in[\widetilde{\delta}, \widetilde{\Delta}]}(1-\gamma) s+\bar{\alpha}_{V} \circ \alpha_{W}^{-1} \circ\left(\frac{\mathbb{I}-\alpha_{Y} \circ \bar{\alpha}_{Y}^{-1}}{\gamma}\right)^{(d)}(s)$

Clearly, $\Upsilon(s, \gamma, d) \leq \psi(\gamma, d)$ for any $s \in[\widetilde{\delta}, \widetilde{\Delta}]$ in view of the definition of $\Upsilon$, and recall from Theorem 1 that $\Upsilon(s, \gamma, d) \geq 0$, thus $0 \leq \Upsilon(s, \gamma, d) \leq \psi(\gamma, d)$. As explained in the proof of Theorem $1, \mathbb{I}-\alpha_{Y} \circ \bar{\alpha}_{Y}^{-1}<\mathbb{I}$ on $\mathbb{R}_{>0}$, hence $\psi(\gamma, d) \rightarrow 0$ as $(\gamma, d) \rightarrow(1, \infty)$. Thus, from the definition of the limit, there exists a pair $\left(\gamma^{*}, d^{*}\right)$ in $(0,1) \times \mathbb{Z}_{>0}$ such that $\psi(\gamma, d)<$ $\frac{1}{2} \widetilde{\alpha}_{Y}(\widetilde{\delta})$ for any $(\gamma, d) \in\left(\gamma^{*}, 1\right] \times\left(d^{*}, \infty\right)$. As a result, for any $s \in[\widetilde{\delta}, \widetilde{\Delta}], \gamma \in\left(\gamma^{*}, 1\right]$ and $d \in\left(d^{*}, \infty\right), \Upsilon(s, \gamma, d) \leq$ $\psi(\gamma, d)<\frac{1}{2} \widetilde{\alpha}_{Y}(\widetilde{\delta})$. Meanwhile, the right hand side of (34) is bounded below by $\frac{1}{2} \widetilde{\alpha}_{Y}(\widetilde{\delta})$, since $\widetilde{\alpha}_{Y} \in \mathcal{K}_{\infty}, s \in[\widetilde{\delta}, \widetilde{\Delta}]$ and $\gamma \in(0,1]$, thus $\Upsilon(s, \gamma, d) \leq \frac{1}{2} \widetilde{\alpha}_{Y}(\widetilde{\delta}) \leq\left(1-\frac{\gamma}{2}\right) \widetilde{\alpha}_{Y}(s)$ and (34) holds.

In view of item (ii) of Theorem 1 and since $\alpha_{Y}(\sigma(x)) \geq$ $\alpha_{Y} \circ \bar{\alpha}_{Y}^{-1}\left(Y_{\gamma, d}(x)\right)$ according to item (i) of Theorem 1 ,

$$
Y_{\gamma, d}(v)-Y_{\gamma, d}(x) \leq \frac{1}{\gamma}\left(-\widetilde{\alpha}_{Y}\left(Y_{\gamma, d}(x)\right)+\Upsilon\left(Y_{\gamma, d}(x), \gamma, d\right)\right) \text {. }
$$

Since $\sigma(x) \leq \Delta, Y_{\gamma, d}(x) \leq \bar{\alpha}_{Y}(\sigma(x)) \leq \bar{\alpha}_{Y}(\Delta)=\widetilde{\Delta}$. Therefore, when $Y_{\gamma, d}(x) \geq \widetilde{\widetilde{\delta}}$, we derive from (34) that $-\widetilde{\alpha}_{Y}\left(Y_{\gamma, d}(x)\right)+\Upsilon\left(Y_{\gamma, d}(x), \gamma, d\right) \leq-\frac{\gamma}{2} \widetilde{\alpha}_{Y}\left(Y_{\gamma, d}(x)\right)$. Thus, from (35),

$$
\begin{aligned}
Y_{\gamma, d}(v) & -Y_{\gamma, d}(x) \\
\leq & \frac{1}{\gamma}\left(-\widetilde{\alpha}_{Y}\left(Y_{\gamma, d}(x)\right)+\Upsilon\left(Y_{\gamma, d}(x), \gamma, d\right)\right) \\
\leq & -\frac{1}{2} \widetilde{\alpha}_{Y}\left(Y_{\gamma, d}(x)\right) .
\end{aligned}
$$

Consider now $Y_{\gamma, d}(x) \in[0, \widetilde{\delta})$. From the definition of $\Upsilon$, note that $\Upsilon(\cdot, \gamma, d)-(1-\gamma) \mathbb{I} \in \mathcal{K}_{\infty}$ or zero. It follows then that $\gamma \mathbb{I}-\widetilde{\alpha}_{Y}+\Upsilon(\cdot, \gamma, d) \in \mathcal{K}_{\infty}$. Indeed, $\gamma \mathbb{I}-\widetilde{\alpha}_{Y}+\Upsilon(\cdot, \gamma, d)=\gamma \mathbb{I}-$

${ }^{5}$ Note since $\mathbb{I}-\bar{\alpha}_{Y}=\mathbb{I}-\alpha_{Y} \circ\left(\bar{\alpha}_{V}+\bar{\alpha}_{W}\right)^{-1} \in \mathcal{K}_{\infty}$ as assumed without loss of generality in Remark 2. Thus $\mathbb{I}-\frac{\bar{\alpha}_{Y}}{2}=\mathbb{I}-\bar{\alpha}_{Y}+\frac{\bar{\alpha}_{Y}}{2} \in \mathcal{K}_{\infty}$, hence $\left(\mathbb{I}-\frac{\bar{\alpha}_{Y}}{2}\right)^{-1} \in \mathcal{K}_{\infty}$ and $\widetilde{\delta}$ is well defined. 
$\widetilde{\alpha}_{Y}+\Upsilon(\cdot, \gamma, d)-(1-\gamma) \mathbb{I}+(1-\gamma) \mathbb{I}=\mathbb{I}-\widetilde{\alpha}_{Y}+\Upsilon(\cdot, \gamma, d)-(1-$ $\gamma) \mathbb{I}$, which is in $\mathcal{K}_{\infty}$ since $\mathbb{I}-\widetilde{\alpha}_{Y} \in \mathcal{K}_{\infty}$ as assumed without loss of generality in Remark 2 and $\Upsilon(\cdot, \gamma, d)-(1-\gamma) \mathbb{I} \in \mathcal{K}_{\infty}$ or zero as noted before. Therefore, for $Y_{\gamma, d}(x) \in[0, \delta)$ and in view of (35),

$$
\begin{aligned}
Y_{\gamma, d}(v) & \leq \frac{1}{\gamma}\left(\gamma Y_{\gamma, d}(x)-\widetilde{\alpha}_{Y}\left(Y_{\gamma, d}(x)\right)+\Upsilon\left(Y_{\gamma, d}(x), \gamma, d\right)\right) \\
Y_{\gamma, d}(v) & \leq \frac{1}{\gamma}\left(\gamma \widetilde{\delta}-\widetilde{\alpha}_{Y}(\widetilde{\delta})+\Upsilon(\widetilde{\delta}, \gamma, d)\right) .
\end{aligned}
$$

From (34), we derive

$$
\begin{aligned}
Y_{\gamma, d}(v) & \leq \frac{1}{\gamma}\left(\gamma \widetilde{\delta}-\widetilde{\alpha}_{Y}(\widetilde{\delta})+\left(1-\frac{\gamma}{2}\right) \widetilde{\alpha}_{Y}(\widetilde{\delta})\right) \\
Y_{\gamma, d}(v) & \leq \frac{1}{\gamma}\left(\gamma \widetilde{\delta}-\frac{\gamma}{2} \widetilde{\alpha}_{Y}(\widetilde{\delta})\right) \\
Y_{\gamma, d}(v) & \leq \widetilde{\delta}-\frac{\widetilde{\alpha}_{Y}(\widetilde{\delta})}{2} .
\end{aligned}
$$

Given the definition of $\widetilde{\delta}$,

$$
Y_{\gamma, d}(v) \leq\left(\mathbb{I}-\frac{\widetilde{\alpha}_{Y}}{2}\right)(\widetilde{\delta})=\underline{\alpha}_{Y}(\delta) .
$$

Thus, whenever $Y_{\gamma, d}(x) \leq \underline{\alpha}_{Y}(\delta), Y_{\gamma, d}(v) \leq \underline{\alpha}_{Y}(\delta)$ follows. Indeed, if $Y_{\gamma, d}(x) \in[\widetilde{\delta}, \widetilde{\Delta}], Y_{\gamma, d}(v) \leq Y_{\gamma, d}(x) \leq \underline{\alpha}_{Y}(\delta)$ according to (36), and if $Y_{\gamma, d}(x) \in[0, \widetilde{\delta})$, we deduce $Y_{\gamma, d}(v) \leq \underline{\alpha}_{Y}(\delta)$ according to (37). Hence the set $\left\{z \in \mathbb{R}^{n}\right.$ : $\left.Y_{\gamma, d}(z) \leq \underline{\alpha}_{Y}(\delta)\right\}$ is forward invariant ${ }^{6}$ for system (5).

We then invoke the same arguments as in the proof of Theorem 2 in [21]. Hence, there exists $\widetilde{\beta} \in \mathcal{K} \mathcal{L}$ such that for any $k \in \mathbb{Z}_{\geq 0}$ and solution $\phi(k, x)$ to system (5),

$$
Y_{\gamma, d}(\phi(k, x)) \leq \max \left\{\widetilde{\beta}\left(Y_{\gamma, d}(x), k\right), \underline{\alpha}_{Y}(\delta)\right\} .
$$

Finally, using $\underline{\alpha}_{Y}(\sigma(x)) \leq Y_{\gamma, d}(x) \leq \bar{\alpha}_{Y}(\sigma(x))$, we obtain

$$
\sigma(\phi(k, x)) \leq \max \left\{\underline{\alpha}_{Y}^{-1}\left(\widetilde{\beta}\left(\bar{\alpha}_{Y}(\sigma(x)), k\right)\right), \delta\right\} .
$$

Thus (11) holds with $\beta(s, k)=\underline{\alpha}_{Y}^{-1}\left(\widetilde{\beta}\left(\bar{\alpha}_{Y}(s), k\right)\right)$.

\section{Proof of Lemma 1}

The proof consists in showing that the conditions of Theorem 1.17 in [22] are satisfied by $V_{\gamma, d}$. Let $\gamma \in(0,1]$, and $d \in \mathbb{Z}_{>0}$. Since $\mathcal{U}(x)$ is non-empty for all $x$ and $J_{\gamma, d}$ is a finite sum, $J_{\gamma, d}$ is trivially finite for all $x$ and $\boldsymbol{u}, J_{\gamma, d}$ is thus a proper function according to the definition in Section 1.A of [22]. Note that $x \mapsto J_{\gamma, d}(x, \boldsymbol{u})$ is a well defined map since $\boldsymbol{u}$ is an admissible sequence for any $x \in \mathbb{R}^{n}$ due to item (ii) of Assumption 2. Moreover, $J_{\gamma, d}$ is simply the composition, multiplication and addition of $f$ and $\ell$, which are continuous functions from item (i) of Assumption 2, thus it follows that $J_{\gamma, d}$ is also a continuous function in $x$ and $\boldsymbol{u}$. Since item (iii) of Assumption 2 holds, $J_{\gamma, d}$ is level-bounded in $\boldsymbol{u}$ locally uniformly to $x$ according to Definition 1.16 in [22]. That is, for each $x \in \mathbb{R}^{n}$ and $a \in \mathbb{R}_{>0}$ there is a neighborhood $\mathcal{X}$ of $x$ such that the set $\left\{(x, \boldsymbol{u}): x \in \mathcal{X}, J_{\gamma, d}(x, \boldsymbol{u}) \leq a\right\}$ is bounded in $\mathbb{R}^{n} \times \mathbb{R}^{m}$. Finally, SA guarantees the existence of

\footnotetext{
${ }^{6}$ The corresponding step was omitted in the proof of Theorem 2 in [21]
}

$\boldsymbol{u}_{\gamma, d}^{*}(x)$, it follows from item (i) of Assumption 2 that with a fixed sequence $\boldsymbol{u}:=\boldsymbol{u}_{\gamma, d}^{*}(x), J_{\gamma, d}(\bar{x}, \boldsymbol{u})$ is continuous in $\bar{x}$. Altogether, we deduce from that $V_{\gamma, d}$ is a continuous function by invoking item (c) of Theorem 1.17 in [22].

\section{Proof of Corollary 1}

Let $\Delta>0, \gamma \in\left(\gamma^{*}, 1\right]$ and $d \in\left(d^{*}, \infty\right], x \in \mathbb{R}^{n}$ be such that $\sigma(x) \leq \Delta$ and $v \in F_{\gamma, d}^{*}(x)$. Since Assumption 1 holds, we can apply the conclusions of Theorem 1. From item (b) of Theorem 1, $Y_{\gamma, d}(v)-Y_{\gamma, d}(x) \leq \frac{1}{\gamma}\left(-\alpha_{W}(\sigma(x))+\right.$ $\left.\Upsilon\left(Y_{\gamma, d}(x), \gamma, d\right)\right)$, where ${ }^{7} \Upsilon(s, \gamma, d)=(1-\gamma) s+\gamma^{d} \bar{\alpha}_{V} \circ$ $\alpha_{W}^{-1} \circ\left(\frac{\mathbb{I}-\alpha_{W} \circ \bar{\alpha}_{Y}^{-1}}{\gamma}\right)^{(d)}(s)$ since $\underline{\alpha}_{Y}=\alpha_{Y}=\alpha_{W}$. We use the following strategy. First, we show that $Y_{\gamma, d}(v)-Y_{\gamma, d}(x) \leq$ $-\epsilon Y_{\gamma, d}(x)$ holds for some $\epsilon>0$ when $Y_{\gamma, d}(x) \in\left[0, a_{Y} L\right]$ due to (13). Then, we show that $Y_{\gamma, d}(v)-Y_{\gamma, d}(x) \leq$ $-\frac{1}{2} \widetilde{\alpha}_{Y}\left(Y_{\gamma, d}(x)\right)$ holds for $\widetilde{\alpha}_{Y}=\alpha_{W} \circ \bar{\alpha}_{Y}^{-1}$ when $Y_{\gamma, d}(x) \in$ $\left(a_{Y} L, \bar{\alpha}_{Y}(\Delta)\right]$ due to (14). To conclude, we combine the two inequalities and we defer to the proof of Theorem 2.

We first derive properties based on the sublinear conditions of Corollary 1. Since $\bar{\alpha}_{V}(s) \leq \bar{a}_{V} \cdot s$ and $\bar{\alpha}_{W}(s) \leq \bar{a}_{W} \cdot s$ for $s \in[0, L], \bar{\alpha}_{Y}(s)=\bar{\alpha}_{V}(s)+\bar{\alpha}_{W}(s) \leq\left(\bar{a}_{V}+\bar{a}_{W}\right) \cdot s=\bar{a}_{Y} \cdot s$. Thus $s=\bar{\alpha}_{Y}^{-1}\left(\bar{\alpha}_{Y}(s)\right) \leq \bar{\alpha}_{Y}^{-1}\left(\bar{a}_{Y} \cdot s\right)$ for $s \in[0, L]$ and, for $s^{\prime}=\bar{a}_{Y} \cdot s \in\left[0, \bar{a}_{Y} L\right]$

$$
\frac{s^{\prime}}{\bar{a}_{Y}} \leq \bar{\alpha}_{Y}^{-1}\left(s^{\prime}\right) .
$$

Similarly, from $a_{W} \cdot s \leq \alpha_{W}(s)$ for $s \in[0, L]$ and taking $s^{\prime}=\alpha_{W}(s) \in\left[0, \alpha_{W}(L)\right]$, we derive that

$$
\alpha_{W}^{-1}\left(s^{\prime}\right) \leq \frac{s^{\prime}}{a_{W}} .
$$

By composing both sides of (40) with $\alpha_{W}$, we obtain that $\alpha_{W}\left(\frac{s}{\bar{a}_{Y}}\right) \leq \alpha_{W} \circ \bar{\alpha}_{Y}^{-1}(s)$ for $s \in\left[0, \bar{a}_{Y} L\right]$, and since $\frac{s}{\bar{a}_{Y}} \in$ $[0, L], a_{W} \frac{s}{\bar{a}_{Y}} \leq \alpha_{W}\left(\frac{s}{\bar{a}_{Y}}\right)$, thus

$$
\frac{a_{W}}{\bar{a}_{Y}} s \leq \alpha_{W} \circ \bar{\alpha}_{Y}^{-1}(s) .
$$

Therefore $s-\alpha_{W} \circ \bar{\alpha}_{Y}^{-1}(s) \leq\left(1-\frac{a_{W}}{\bar{a}_{Y}}\right) s$, and, for any $s \in$ $\left[0, \bar{a}_{Y} L\right]$,

$$
\left(\frac{s-\alpha_{W} \circ \bar{\alpha}_{Y}^{-1}(s)}{\gamma}\right) \leq \gamma^{-1}\left(1-\frac{a_{W}}{\bar{a}_{Y}}\right) s .
$$

On the other hand, according to (13), $1-\gamma^{*}<\frac{a_{W}}{\bar{a}_{Y}}$, thus $1-\frac{a_{W}}{\bar{a}_{Y}}<\gamma^{*}$. Since $\gamma^{*}<\gamma, 1-\frac{a_{W}}{\bar{a}_{Y}}<\gamma$. With this inequality, we derive from (43) that $\left(\frac{s-\alpha_{W} \circ \bar{\alpha}_{Y}^{-1}(s)}{\gamma}\right) \leq s$ for any $s \in\left[0, \bar{a}_{Y} L\right]$. Thus $\left(\frac{s-\alpha_{W} \circ \bar{\alpha}_{Y}^{-1}(s)}{\gamma}\right) \in\left[0, \bar{a}_{Y} L\right]$ for $s \in\left[0, \bar{a}_{Y} L\right]$. We can then apply the first inequality in (43) iteratively and obtain $\left(\frac{\mathbb{I}-\alpha_{W} \circ \bar{\alpha}_{Y}^{-1}}{\gamma}\right)^{(d)}(s) \leq \gamma^{-d}\left(1-\frac{a_{W}}{\bar{a}_{Y}}\right)^{d} s$ for $s \in\left[0, \bar{a}_{Y} L\right]$. Also from (43), $\gamma^{*}<\gamma$ and $d>d^{*}$, we have that $\left(\frac{1-\frac{a_{W}}{\bar{a}_{Y}}}{\gamma^{*}}\right)^{d^{*}}>\left(\frac{1-\frac{a_{W}}{\bar{a}_{Y}}}{\gamma^{*}}\right)^{d}>\gamma^{-d}\left(1-\frac{a_{W}}{\bar{a}_{Y}}\right)^{d}$. Hence, according to (12) and (43), for $s \in\left[0, \bar{a}_{Y} L\right]$,

$$
\left(\frac{\mathbb{I}-\alpha_{W} \circ \bar{\alpha}_{Y}^{-1}}{\gamma}\right)^{(d)}(s) \leq \gamma^{-d}\left(1-\frac{a_{W}}{\bar{a}_{Y}}\right)^{d} s \leq \alpha_{W}(L) .
$$

\footnotetext{
${ }^{7}$ The case when $\gamma=1$ or $d=\infty$ will be discussed later.
} 
Since (44) holds, we can invoke (41) and obtain for $s \in$ $\left[0, \bar{a}_{Y} L\right]$

$$
\alpha_{W}^{-1} \circ\left(\frac{\mathbb{I}-\alpha_{W} \circ \bar{\alpha}_{Y}^{-1}}{\gamma}\right)^{(d)}(s) \leq \frac{\gamma^{-d}}{a_{W}}\left(1-\frac{a_{W}}{\bar{a}_{Y}}\right)^{d} s .
$$

Moreover, from (44), it follows that, for $s \in\left[0, \bar{a}_{Y} L\right]$,

$$
\alpha_{W}^{-1} \circ\left(\frac{\mathbb{I}-\alpha_{W} \circ \bar{\alpha}_{Y}^{-1}}{\gamma}\right)^{(d)}(s) \leq \alpha_{W}^{-1} \circ \alpha_{W}(L)=L .
$$

From (45) and (46), we conclude that $\gamma^{d} \bar{\alpha}_{V} \circ \alpha_{W}^{-1} \circ$ $\left(\frac{\mathbb{I}-\alpha_{W} \circ \bar{\alpha}_{Y}^{-1}}{\gamma}\right)^{(d)}(s) \leq \gamma^{d} \bar{a}_{V} \cdot \alpha_{W}^{-1} \circ\left(\frac{\mathbb{I}-\alpha_{W} \circ \bar{\alpha}_{Y}^{-1}}{\gamma}\right)^{(d)}(s) \leq$ $\gamma^{d} \bar{a}_{V} \frac{\gamma^{-d}}{a_{W}}\left(1-\frac{a_{W}}{\bar{a}_{Y}}\right)^{d} s=\frac{\bar{a}_{V}}{a_{W}}\left(1-\frac{a_{W}}{\bar{a}_{Y}}\right)^{d} s$ for $s \in\left[0, \bar{a}_{Y} L\right]$ holds. Therefore, for $s \in\left[0, \bar{a}_{Y} L\right]$,

$$
\Upsilon(s, \gamma, d) \leq(1-\gamma) s+\frac{\bar{a}_{V}}{a_{W}}\left(1-\frac{a_{W}}{\bar{a}_{Y}}\right)^{d} s .
$$

Note that (47) holds even for $\gamma=1$ or $d=\infty$, indeed $\Upsilon(s, 1, d) \leq \frac{\bar{a}_{V}}{a_{W}}\left(1-\frac{a_{W}}{\bar{a}_{Y}}\right)^{d} s$ and $\Upsilon(s, \gamma, \infty)=(1-\gamma) s$ for $s \in\left[0, \bar{a}_{Y} L\right]$.

Since $-\alpha_{W}(\sigma(x)) \leq-\alpha_{W} \circ \bar{\alpha}_{Y}^{-1}\left(Y_{\gamma, d}(x)\right)$ holds from item (i) of Theorem 1, for $Y_{\gamma, d}(x) \in\left[0, \bar{a}_{Y} L\right]$ we derive that $-\alpha_{W}(\sigma(x)) \leq-\frac{a_{W}}{\bar{a}_{Y}} Y_{\gamma, d}(x)$ from (42). Applying this inequality and (47) to item (ii) of Theorem 1, we find $Y_{\gamma, d}(v)-Y_{\gamma, d}(x) \leq \frac{-\frac{a_{W}}{\bar{a}_{Y}}+(1-\gamma)+\frac{\bar{a}_{V}}{a_{W}}\left(1-\frac{a_{W}}{\bar{a}_{Y}}\right)^{d}}{\gamma} Y_{\gamma, d}(x)$. For $\left(\gamma^{*}, d^{*}\right)$ as defined in (13), and since $\gamma \in\left(\gamma^{*}, 1\right]$ and $d>d^{*}$, it follows

$$
0<1-\gamma+\frac{\bar{a}_{V}}{a_{W}}\left(1-\frac{a_{W}}{\bar{a}_{Y}}\right)^{d}<\frac{a_{W}}{\bar{a}_{Y}} .
$$

Consequently, there exist $\epsilon \in\left(0, \frac{a_{W}}{\bar{a}_{Y}}\right)$ such that $-\frac{a_{W}}{\bar{a}_{Y}}+1-$ $\gamma+\frac{\bar{a}_{V}}{a_{W}}\left(1-\frac{a_{W}}{\bar{a}_{Y}}\right)^{d}<-\frac{\epsilon}{\gamma}<-\epsilon$. Finally, we conclude that $Y_{\gamma, d}(v)-Y_{\gamma, d}(x) \leq-\epsilon Y_{\gamma, d}(x)$ for $Y_{\gamma, d}(x) \in\left[0, \bar{a}_{Y} L\right]$ since (13) holds.

For $Y_{\gamma, d}(x) \in\left(\bar{a}_{Y} L, \bar{\alpha}_{Y}(\Delta)\right]$, the existence of $\gamma^{*}$ and $d^{*}$ such that (14) holds follows from the same arguments as for the existence of $\gamma^{*}$ and $d^{*}$ such that (34) holds in the proof of Theorem 2, with $\widetilde{\Delta}:=\bar{\alpha}_{Y}(\Delta)$ and $\widetilde{\delta}:=\bar{a}_{Y} L$. By following the steps of the proof of Theorem 2 for (36) we obtain that $Y_{\gamma, d}(v)-Y_{\gamma, d}(x)<-\frac{1}{2} \widetilde{\alpha}_{Y}\left(Y_{\gamma, d}(x)\right)$ for $Y_{\gamma, d}(x) \in$ $\left(\bar{a}_{Y} L, \bar{\alpha}_{Y}(\Delta)\right]$.

We have found that $Y_{\gamma, d}(v)-Y_{\gamma, d}(x)$ decreases for all $Y_{\gamma, d}(x) \in\left(0, \bar{\alpha}_{Y}(\Delta)\right]$. In particular, by $-\epsilon Y_{\gamma, d}(x)$ for $Y_{\gamma, d}(x) \in\left[0, \bar{a}_{Y} L\right]$ and by $-\frac{1}{2} \widetilde{\alpha}_{Y}\left(Y_{\gamma, d}(x)\right)$ for $Y_{\gamma, d}(x) \in$ $\left(\bar{a}_{Y} L, \bar{\alpha}_{Y}(\Delta)\right]$. We conclude the proof by noting that $Y_{\gamma, d}(v)-$ $Y_{\gamma, d}(x) \leq-\min \left\{\epsilon \mathbb{I}, \frac{1}{2} \widetilde{\alpha}_{Y}\right\}\left(Y_{\gamma, d}(x)\right)$. The desired result is then derived by following the final steps of Theorem 2 .

\section{E. Sketch of proof of Corollary 2}

Let $x \in \mathbb{R}^{n}, v \in F_{\gamma, d}^{*}(x)$ and $\gamma \in\left(\gamma^{*}, 1\right], d \in\left(d^{*}, \infty\right]$. Since Assumption 1 holds with Corollary 1 conditions with $L=\infty$, we can use the sublinear developments of Corollary 1 everywhere, that is, for all $x \in \mathbb{R}^{n}$. In particular, we have shown that given (13), there exists $\epsilon \in\left(0, \frac{a_{W}}{\bar{a}_{Y}}\right)$ such that $Y_{\gamma, d}(v)-Y_{\gamma, d}(x) \leq-\epsilon Y_{\gamma, d}(x)$ holds for $Y_{\gamma, d}(x) \in\left[0, \bar{a}_{Y} L\right]$.
Similarly in Corollary 2 case, we derive from (15) that $Y_{\gamma, d}(v)-Y_{\gamma, d}(x) \leq-\epsilon Y_{\gamma, d}(x)$ holds for any $Y_{\gamma, d}(x) \in \mathbb{R}_{\geq 0}$. We now proceed with the same argument as the proof of Corollary 2 in [21]. Let $x \in \mathbb{R}^{n}$ and denote $\phi(k, x)$ be a corresponding solution to (5) at time $k \in \mathbb{Z}_{\geq 0}$, it holds that $Y_{\gamma, d}(\phi(k, x)) \leq(1-\epsilon)^{k} Y_{\gamma, d}(x)$. Since $Y_{\gamma, d}(x) \geq$ $\alpha_{W}(\sigma(x)) \geq a_{W} \sigma(x)$ and $Y_{\gamma, d}(x) \leq \bar{\alpha}_{Y}(\sigma(x)) \leq \bar{a}_{Y} \sigma(x)$, we conclude that Corollary 2 holds with $K=\frac{\bar{a}_{Y}}{a_{W}}=\frac{\bar{a}_{V}+\bar{a}_{W}}{a_{W}}$ and $\lambda=-\ln (1-\epsilon)$.

\section{F. Proof of Lemma 2}

Item (ii) of Lemma 2 is derived immediately by substitution, since $\bar{d}=\frac{\ln \left(\bar{a}_{V}\left(\bar{a}_{V}+\bar{a}_{W}\right) / a_{W}^{2}\right)}{-\ln \left(1-\bar{a}_{V} \bar{a}_{W}\right)}$ and $d_{[6]}:=\frac{\bar{a}_{V}\left(\bar{a}_{V}+\bar{a}_{W}\right)}{a_{W}^{2}}$. Item (i) of Lemma 2 follows since $\frac{\bar{\gamma}}{\gamma_{[21]}}<1$ implies $\frac{\left(\bar{a}_{V}+\bar{a}_{W}-a_{W}\right)\left(\bar{a}_{V}+a_{W}\right)}{\left(\bar{a}_{V}+\bar{a}_{W}\right) \bar{a}_{V}}=\frac{\bar{a}_{V}^{2}+\bar{a}_{V} \bar{a}_{W}+a_{W} \bar{a}_{W}-a_{W}^{2}}{\bar{a}_{V}^{2}+\bar{a}_{V} \bar{a}_{W}}<1$, which in turn implies $a_{W} \bar{a}_{W}<a_{W}^{2}$. Item (i) of Lemma 2 holds since $a_{W}>0$ by assumption of Corollary 2 .

\section{G. Proof of Theorem 3}

Let $x \in \mathbb{R}^{n}, \gamma \in(0,1], d \in \mathbb{Z}_{>0}$. According to SA, the input sequence $\left[u_{0}^{*}, \ldots, u_{d-1}^{*}, u_{d}^{*}\right]=\boldsymbol{u}_{\gamma, d}^{*}(x)$ exists and $J_{\gamma, d}\left(x, \boldsymbol{u}_{\gamma, d}^{*}(x)\right)=V_{\gamma, d}(x)$. The lower bounds on $V_{\gamma, \infty}(x)$ and $V_{1, d}(x)$ in (16a) and (16b), respectively, follow from the definitions of $V_{\gamma, \infty}(x)$ and $V_{1, d}(x)$ in (3). The other inequalities are proved in the following.

Define $\phi_{k}^{*}:=\phi\left(k, x,\left.\boldsymbol{u}_{\gamma, d}^{*}(x)\right|_{k}\right)$ a solution to (1) at time $k \in\{0, \ldots, d\}$, initialized at $x$, and define $\ell_{k}^{*}:=\ell\left(\phi_{k}^{*}, u_{k}^{*}\right)$ the corresponding stage cost at time $k$. To prove the second inequality in equation (16a), consider the infinite sequence $\hat{\boldsymbol{u}}:=\left[u_{0}^{*}, \ldots, u_{d-1}^{*}, \boldsymbol{u}_{\gamma, \infty}^{*}\left(\phi_{d}^{*}\right)\right]$, where $\boldsymbol{u}_{\gamma, \infty}^{*}$ exist according to SA. By definition of $V_{\gamma, \infty}(x)$ and $V_{\gamma, d}(x)$, $V_{\gamma, \infty}(x) \leq J_{\gamma, \infty}(x, \hat{\boldsymbol{u}})=J_{\gamma, d-1}\left(x,\left[u_{0}^{*}, \ldots, u_{d-1}^{*}\right]\right)+$ $\gamma^{d} J_{\gamma, \infty}\left(\phi_{d}^{*}, \boldsymbol{u}_{\gamma, \infty}^{*}\left(\phi_{d}^{*}\right)\right) \leq V_{\gamma, d}(x)+\gamma^{d} V_{\gamma, \infty}\left(\phi_{d}^{*}\right)$. According to item (i) of Assumption 1, $V_{\gamma, \infty}\left(\phi_{d}^{*}\right) \leq \bar{\alpha}_{V}\left(\sigma\left(\phi_{d}^{*}\right)\right)$. To bound $\sigma\left(\phi_{d}^{*}\right)$, we invoke (79) in the appendix. Hence, $V_{\gamma, \infty}\left(\phi_{d}^{*}\right) \leq$ $\bar{\alpha}_{V} \circ \underline{\alpha}_{Y}^{-1} \circ\left(\frac{\mathbb{I}-\alpha_{Y} \circ \bar{\alpha}_{Y}^{-1}}{\gamma}\right)^{(d)}\left(Y_{\gamma, d}(x)\right)$. By invoking item (i) of Theorem 1, $Y_{\gamma, d}(x) \leq \bar{\alpha}_{Y}(\sigma(x))$ and we obtain the second inequality of (16a).

For (16b), consider the finite sequence $\boldsymbol{u}_{\gamma, d}^{*}(x)$. Note that

$$
V_{1, d}(x) \leq J_{1, d}\left(x, \boldsymbol{u}_{\gamma, d}^{*}(x)\right)=\sum_{k=0}^{d} \ell_{k}^{*}
$$

By applying Bellman principle to $V_{\gamma, d}(x)$, as done in the proof of Theorem 5, it follows that $V_{\gamma, d-k}\left(\phi_{k}^{*}\right)=\ell_{k}^{*}+$ $\gamma V_{\gamma, d-(k+1)}\left(\phi_{k+1}^{*}\right)$ for $k \in\{0, \ldots, d-1\}$ and $V_{\gamma, 0}\left(\phi_{d}^{*}\right)=\ell_{d}^{*}$. By summation we have $\sum_{k=0}^{d} V_{\gamma, d-k}\left(\phi_{k}^{*}\right)=\sum_{k=0}^{d} \ell_{k}^{*}+$ $\gamma \sum_{k=0}^{d-1} V_{\gamma, d-(k+1)}\left(\phi_{k+1}^{*}\right) V_{\gamma, d}\left(\phi_{0}^{*}\right)+\sum_{k=1}^{d} V_{\gamma, d-k}\left(\phi_{k}^{*}\right)=$ $\sum_{k=0}^{d} \ell_{k}^{*}+\gamma \sum_{k=1}^{d} V_{\gamma, d-k}\left(\phi_{k}^{*}\right)$. Since $\phi_{0}^{*}=x$,

$$
V_{\gamma, d}(x)+(1-\gamma) \sum_{k=1}^{d} V_{\gamma, d-k}\left(\phi_{k}^{*}\right)=\sum_{k=0}^{d} \ell_{k}^{*} .
$$

Therefore, in view of (49), $V_{1, d}(x) \leq V_{\gamma, d}(x)+(1-$ $\gamma) \sum_{k=1}^{d} V_{\gamma, d-k}\left(\phi_{k}^{*}\right)$. The proof is completed by noting that 
$V_{\gamma, d-k}\left(\phi_{k}^{*}\right) \leq \bar{\alpha}_{V}\left(\sigma\left(\phi_{k}^{*}\right)\right) \leq v_{\gamma, k}(x):=\bar{\alpha}_{V} \circ \underline{\alpha}_{Y}^{-1} \circ$ $\left(\frac{\mathbb{I}-\alpha_{Y} \circ \bar{\alpha}_{Y}^{-1}}{\gamma}\right)^{(k)} \circ \bar{\alpha}_{Y}(\sigma(x))$, which holds in view of (79).

\section{H. Proof of Lemma 3}

Let $\Delta>0, x \in\left\{z \in \mathbb{R}^{n}: \sigma(z) \leq \Delta\right\}$ and $\widetilde{\Delta}=$ $\bar{\alpha}_{Y}(\Delta)$. When $\gamma=1$, it follows that $v_{1, d}(x)=\bar{\alpha}_{V} \circ \underline{\alpha}_{Y}^{-1} \circ$ $\left(\mathbb{I}-\alpha_{Y} \circ \bar{\alpha}_{Y}^{-1}\right)^{(d)} \circ \bar{\alpha}_{Y}(\sigma(x))$, which can be made as small as desired by noting that $\left(\mathbb{I}-\alpha_{Y} \circ \bar{\alpha}_{Y}^{-1}\right)^{(d)}$ is decreasing in $d$, as explained in the proof of Theorem 1. Thus $v_{1, d}(x) \rightarrow 0$ when $d \rightarrow \infty$.

When $\gamma \in\left[1-\frac{\alpha_{Y}(\Delta)}{\bar{\alpha}_{Y}(\Delta)}, 1\right)$, it follows that $(1-\gamma) \bar{\alpha}_{Y}(\Delta) \leq$ $\alpha_{Y}(\Delta)$, thus $(1-\gamma) \widetilde{\Delta} \leq \alpha_{Y} \circ \bar{\alpha}_{Y}^{-1}(\widetilde{\Delta})$ by definition of $\widetilde{\Delta}$, and hence $\frac{\widetilde{\Delta}-\alpha_{Y} \circ \bar{\alpha}_{Y}^{-1}(\widetilde{\Delta})}{\gamma} \leq \widetilde{\Delta}$. We have obtained that $\left(\frac{\mathbb{I}-\alpha_{Y} \circ \bar{\alpha}_{Y}^{-1}}{\gamma}\right)^{(d)}(\widetilde{\Delta}) \leq \widetilde{\Delta}$. On the other hand, since $\sigma(x) \leq \Delta$ and $\frac{\mathbb{I}-\alpha_{Y} \circ \bar{\alpha}_{Y}^{-1}}{\gamma}, \bar{\alpha}_{Y} \in \mathcal{K}_{\infty}$ as assumed without loss of generality in Remark 2 and Theorem 1 respectively, we have $\frac{\mathbb{I}-\alpha_{Y} \circ \bar{\alpha}_{Y}^{-1}}{\gamma}\left(\bar{\alpha}_{Y}(\sigma(x))\right) \leq \frac{\mathbb{I}-\alpha_{Y} \circ \bar{\alpha}_{Y}^{-1}}{\gamma}(\Delta) \leq \widetilde{\Delta}$. Finally, we have that $\gamma^{d} v_{\gamma, d}(x) \leq \gamma^{d} \bar{\alpha}_{V} \circ \underline{\alpha}_{Y}(\widetilde{\Delta})$, thus $\gamma^{d} v_{\gamma, d}(x) \rightarrow 0$ when $d \rightarrow \infty$.

Since $\gamma^{d} v_{\gamma, d}(x) \rightarrow 0$ for $\gamma \in\left[1-\frac{\alpha_{Y}(\Delta)}{\bar{\alpha}_{Y}(\Delta)}, 1\right]$ when $d \rightarrow \infty$, it follows from the sandwich rule and (16a) that $V_{\gamma, d}(x)+$ $\gamma^{d} v_{\gamma, d}(x) \rightarrow V_{\gamma, \infty}(x)$ when $d \rightarrow \infty$.

\section{Proof of Corollary 3}

Let $d \in \mathbb{Z}_{>0}, \gamma \in(0,1]$ and $x \in \mathbb{R}^{n}$. Item (i) of Corollary 3 follows immediately by application of (16a) and of the conditions of Corollary 2. Indeed, since $v_{d}(x)=\bar{\alpha}_{V} \circ \underline{\alpha}_{Y}^{-1} \circ\left(\frac{\mathbb{I}-\alpha_{Y} \circ \bar{\alpha}_{Y}^{-1}}{\gamma}\right)^{(d)} \circ \bar{\alpha}_{Y}(\sigma(x))$, we have $v_{d}(x) \leq \gamma^{-d} \frac{\bar{a}_{V}\left(\bar{a}_{V}+\bar{a}_{W}\right)}{a_{W}}\left(1-\frac{a_{W}}{\bar{a}_{V}+\bar{a}_{W}}\right)^{d} \sigma(x)$. Thus $V_{\gamma, \infty} \leq V_{\gamma, d}(x)+\gamma^{d} v_{d}(x) \leq V_{\gamma, d}(x)+$ $\frac{\bar{a}_{V}\left(\bar{a}_{V}+\bar{a}_{W}\right)}{a_{W}}\left(1-\frac{a_{W}}{\bar{a}_{V}+\bar{a}_{W}}\right)^{d} \sigma(x)=V_{\gamma, d}(x)+\hat{v}_{d}(x)$, which is the desired result.

Assume now that $\gamma \in\left(1-\frac{a_{W}}{\bar{a}_{V}+\bar{a}_{W}}, 1\right]$. Since $\gamma \in(0,1]$, Theorem 3 holds. Recall the definition of $v_{k}$ from (16b) and notice that $v_{k}(x) \leq \gamma^{-k} \frac{\bar{a}_{V}\left(\bar{a}_{V}+\bar{a}_{W}\right)}{a_{W}}\left(1-\frac{a_{W}}{\bar{a}_{V}+\bar{a}_{W}}\right)^{k} \sigma(x)$, in view of the definition of $v_{k}$ and by direct application of the conditions of Corollary 2. Thus, $\sum_{k=1}^{d} v_{k}(x) \leq \sigma(x) \frac{\bar{a}_{V}\left(\bar{a}_{V}+\bar{a}_{W}\right)}{a_{W}} \sum_{k=1}^{d} \gamma^{-k}\left(1-\frac{a_{W}}{\bar{a}_{V}+\bar{a}_{W}}\right)^{k}=$ $\sigma(x) \frac{\bar{a}_{V}\left(\bar{a}_{V}+\bar{a}_{W}\right)}{a_{W}} \sum_{k=1}^{\infty} \gamma^{-k}\left(1-\frac{a_{W}}{\bar{a}_{V}+\bar{a}_{W}}\right)^{k}$. Since $1-\frac{a_{W}}{\bar{a}_{V}+\bar{a}_{W}}<\gamma$, $\sum_{k=1}^{\infty} \gamma^{-k}\left(1-\frac{a_{W}}{\bar{a}_{V}+\bar{a}_{W}}\right)^{k}=\frac{\gamma^{-1}\left(1-\frac{a_{W}}{\bar{a}_{V}+\bar{a}_{W}}\right)}{1-\gamma^{-1}\left(1-\frac{a_{W}}{\bar{a}_{V}+\bar{a}_{W}}\right)}=\left(1-\frac{a_{W}}{\bar{a}_{V}+\bar{a}_{W}}\right)$ $\frac{\bar{a}_{V}+\bar{a}_{W}}{(1-\gamma)\left(\bar{a}_{V}+\bar{a}_{W}\right)}$. Finally, $(1-\gamma) \sum_{k=1}^{d} v_{k}(x) \leq S_{v}(x)$ where $S_{v}(x):=(1-\gamma) \sigma(x) \frac{\bar{a}_{V}\left(\bar{a}_{V}+\bar{a}_{W}\right)}{a_{W}}\left(1-\frac{a_{W}}{\bar{a}_{V}+\bar{a}_{W}}\right) \frac{\bar{a}_{V}+\bar{a}_{W}}{a_{W}-(1-\gamma)\left(\bar{a}_{V}+\bar{a}_{W}\right)}$ Thus $V_{1, d} \leq V_{\gamma, d}(x)+S_{v}(x)$ and the proof is completed.

\section{J. Proof of Theorem 4}

The proof consist in building a Lyapunov function for the near-optimal cost, and to conclude by following similar steps as in the proof of Theorem 2, by suitable selection of $\epsilon$. Let $x \in \mathbb{R}^{n},(\gamma, d) \in \mathcal{P}$ and $\epsilon>0$. Per Assumption 3, there exists a near-optimal input sequence $\left[\hat{u}_{0}, \hat{u}_{1}, \ldots, \hat{u}_{d}\right]=\hat{\boldsymbol{u}}_{\gamma, d}(x)$, such that $\hat{v}=f\left(x, \hat{u}_{0}\right)$ and $\widehat{V}_{\gamma, d}(x)=J_{\gamma, d}\left(x, \hat{\boldsymbol{u}}_{\gamma, d}(x)\right)$. Let $k \in$ $\{0, \ldots, d\}$ and define $\hat{\phi}_{k}:=\phi\left(k, x,\left.\hat{\boldsymbol{u}}_{\gamma, d}(x)\right|_{k}\right)$ the solution to (1) with input $\hat{\boldsymbol{u}}_{\gamma, d}(x)$ initialized at $x, \hat{\ell}_{k}:=\ell\left(\hat{\phi}_{k}, \hat{u}_{k}\right)$ and $\widehat{V}_{\gamma, d-k}\left(\phi_{k}\right):=J_{\gamma, d-k}\left(\hat{\phi}_{k},\left[\hat{u}_{k}, \ldots, \hat{u}_{d}\right]\right)$.

By definition of $\widehat{V}_{\gamma, d}$ and $\hat{\ell}_{k}$, it follows from (2) that $\widehat{V}_{\gamma, d-k}\left(\hat{\phi}_{k}\right)=\hat{\ell}_{k}+\gamma \widehat{V}_{\gamma, d-(k+1)}\left(\hat{\phi}_{k+1}\right)$ for $k \in\{0, \ldots, d-1\}$ and $\widehat{V}_{\gamma, 0}\left(\hat{\phi}_{d}\right)=\hat{\ell}_{d}$. Invoking item (ii) of Assumption 1, we derive $W\left(\hat{\phi}_{k}\right) \geq-\hat{\ell}_{k}+\alpha_{W}\left(\sigma\left(\hat{\phi}_{k}\right)\right)+W\left(\hat{\phi}_{k+1}\right)$. Thus, given $\widehat{Y}_{\gamma, d-k}\left(\hat{\phi}_{k}\right):=\widehat{V}_{\gamma, d-k}\left(\hat{\phi}_{k}\right)+W\left(\hat{\phi}_{k}\right)$, we derive $\widehat{Y}_{\gamma, d-k}\left(\hat{\phi}_{k}\right) \geq$ $\alpha_{W}\left(\sigma\left(\hat{\phi}_{k}\right)\right)+\gamma \hat{Y}_{\gamma, d-(k+1)}\left(\hat{\phi}_{k+1}\right)+(1-\gamma) W\left(\hat{\phi}_{k+1}\right)$. For $k=d$, we derive similarly that $\widehat{Y}_{\gamma, 0}\left(\hat{\phi}_{d}\right) \geq \alpha_{W}\left(\sigma\left(\hat{\phi}_{d}\right)\right)$, thus for any $k \in\{0, \ldots, d\}$, it follows that

$$
\widehat{Y}_{\gamma, d-k}\left(\hat{\phi}_{k}\right) \geq \alpha_{W}\left(\sigma\left(\hat{\phi}_{k}\right)\right) \text {. }
$$

On the other hand, from $\widehat{Y}_{\gamma, d-k}\left(\hat{\phi}_{k}\right) \geq \alpha_{W}\left(\sigma\left(\hat{\phi}_{k}\right)\right)+$ $\gamma \widehat{Y}_{\gamma, d-(k+1)}\left(\hat{\phi}_{k+1}\right)+(1-\gamma) W\left(\hat{\phi}_{k+1}\right)$ for $k \in\{0, \ldots, d-1\}$ established above, we deduce $\widehat{Y}_{\gamma, d-k}\left(\hat{\phi}_{k}\right) \geq \alpha_{W}\left(\sigma\left(\hat{\phi}_{k}\right)\right)+$ $\gamma \widehat{Y}_{\gamma, d-(k+1)}\left(\hat{\phi}_{k+1}\right)$ since $W \geq 0$, which implies

$$
\widehat{Y}_{\gamma, d-(k+1)}\left(\hat{\phi}_{k+1}\right) \leq \frac{\widehat{Y}_{\gamma, d-k}\left(\hat{\phi}_{k}\right)-\alpha_{W}\left(\sigma\left(\hat{\phi}_{k}\right)\right)}{\gamma} .
$$

We now derive an upper-bound for $\widehat{Y}_{\gamma, d-k}\left(\hat{\phi}_{k}\right)$ in terms of $\widehat{Y}_{\gamma, d}(x)$. Note that $\widehat{Y}_{\gamma, d-(k+1)}\left(\hat{\phi}_{k+1}\right) \leq \frac{\widehat{Y}_{\gamma, d-k}\left(\hat{\phi}_{k}\right)}{\gamma}$ holds. In view of Assumptions 1 and 3, we have that $\widehat{V}_{\gamma, d-k}\left(\hat{\phi}_{k}\right)+$ $W\left(\hat{\phi}_{k}\right) \leq \bar{\alpha}_{V}\left(\sigma\left(\hat{\phi}_{k}\right)\right)+\eta(\epsilon, \gamma, d)+\bar{\alpha}_{W}\left(\sigma\left(\hat{\phi}_{k}\right)\right)$, that is, $\widehat{Y}_{\gamma, d-k}\left(\hat{\phi}_{k}\right) \leq \eta(\epsilon, \gamma, d)+\bar{\alpha}_{Y}\left(\sigma\left(\hat{\phi}_{k}\right)\right)$, where $\bar{\alpha}_{Y}:=$ $\bar{\alpha}_{V}+\bar{\alpha}_{W}$. When $\bar{\alpha}_{Y}\left(\sigma\left(\hat{\phi}_{k}\right)\right) \geq \eta(\epsilon, \gamma, d)$, it follows that $\bar{\alpha}_{Y}^{-1}\left(\frac{\widehat{Y}_{\gamma, d-k}\left(\hat{\phi}_{k}\right)}{2}\right) \leq \sigma\left(\hat{\phi}_{k}\right)$, which implies

$$
\widetilde{\alpha}_{Y}\left(\widehat{Y}_{\gamma, d-k}\left(\hat{\phi}_{k}\right)\right) \leq \alpha_{W}\left(\sigma\left(\hat{\phi}_{k}\right)\right)
$$

where $\widetilde{\alpha}_{Y}(s):=\alpha_{W} \circ \bar{\alpha}_{Y}^{-1}\left(\frac{s}{2}\right)$ for all $s \geq 0$. When $\bar{\alpha}_{Y}\left(\sigma\left(\hat{\phi}_{k}\right)\right) \leq \eta(\epsilon, \gamma, d)$, it follows that

$$
\widehat{Y}_{\gamma, d-k}\left(\hat{\phi}_{k}\right) \leq 2 \eta(\epsilon, \gamma, d) \text {. }
$$

From (52), (53) and (54), we derive $\widehat{Y}_{\gamma, d-(k+1)}\left(\phi_{k+1}\right) \leq$ $\max \left\{\left(\frac{\mathbb{I}-\widetilde{\alpha}_{Y}}{\gamma}\right)\left(\widehat{Y}_{\gamma, d-k}\left(\hat{\phi}_{k}\right)\right), 2 \frac{\eta(\epsilon, \gamma, d)}{\gamma}\right\}$. For the sake of convenience, we introduce parameter $\mu>0$ such that such that $2 \frac{\eta(\epsilon, \gamma, d)}{\gamma} \leq \mu$ and that $1-\frac{\widetilde{\alpha}_{Y}(\mu)}{\mu} \leq \gamma$. Parameter $\mu$ can be introduced without loss of generality, since the first condition $2 \frac{\eta(\epsilon, \gamma, d)}{\gamma} \leq \mu$ can be always verified by taking $\epsilon$ small, and the second is verified by taking $\gamma$ close to 1 . Moreover, we will require later in the proof analogous conditions on $\gamma$ and $\eta$ to guarantee stability. Thus,

$$
\widehat{Y}_{\gamma, d-(k+1)}\left(\phi_{k+1}\right) \leq \max \left\{\left(\frac{\mathbb{I}-\widetilde{\alpha}_{Y}}{\gamma}\right)\left(\widehat{Y}_{\gamma, d-k}\left(\hat{\phi}_{k}\right)\right), \mu\right\} \text {. }
$$

We now show that $\frac{\mathbb{I}-\widetilde{\alpha}_{Y}}{\gamma} \in \mathcal{K}_{\infty}$. Indeed, as explained in Remark 2, we can assume without loss of generality that $\mathbb{I}-\alpha_{W} \circ\left(\bar{\alpha}_{V}+\bar{\alpha}_{W}\right) \in \mathcal{K}_{\infty}$. Thus $2 \mathbb{I}-\alpha_{W} \circ$ $\bar{\alpha}_{Y}^{-1} \in \mathcal{K}_{\infty}$, therefore $\mathbb{I}-\alpha_{W} \circ \bar{\alpha}_{Y}^{-1} \circ \frac{\mathbb{I}}{2}=\mathbb{I}-\widetilde{\alpha}_{Y} \in$ $\mathcal{K}_{\infty}$ and we conclude $\frac{\mathbb{I}-\widetilde{\alpha}_{Y}}{\gamma} \in \mathcal{K}_{\infty}$. Hence, with one iteration of (55) to itself, we obtain $\widehat{Y}_{\gamma, d-(k+2)}\left(\hat{\phi}_{k+2}\right) \leq$ 
$\max \left\{\left(\frac{\mathbb{I}-\widetilde{\alpha}_{Y}}{\gamma}\right)^{(2)}\left(\widehat{Y}_{\gamma, d-k}\left(\hat{\phi}_{k}\right)\right),\left(\frac{\mathbb{I}-\widetilde{\alpha}_{Y}}{\gamma}\right)(\mu), \mu\right\}$. By successive iterations of (55) and noting that $\left(\frac{\mathbb{I}-\widetilde{\alpha}_{Y}}{\gamma}\right)(\mu) \leq \mu$ because $1-\frac{\widetilde{\alpha}_{Y}(\mu)}{\mu} \leq \gamma$, we obtain

$$
\widehat{Y}_{\gamma, 0}\left(\hat{\phi}_{d}\right) \leq \max \left\{\left(\frac{\mathbb{I}-\widetilde{\alpha}_{Y}}{\gamma}\right)^{(d)}\left(\widehat{Y}_{\gamma, d}(x)\right), \mu\right\} .
$$

Since (51) holds,

$$
\sigma\left(\hat{\phi}_{d}\right) \leq \sigma_{d}\left(\widehat{Y}_{\gamma, d}(x)\right),
$$

where $\sigma_{d}\left(\widehat{Y}_{\gamma, d}(x)\right):=\max \left\{\alpha_{W}^{-1} \circ\left(\frac{\mathbb{I}-\widetilde{\alpha}_{Y}}{\gamma}\right)^{(d)}\left(\widehat{Y}_{\gamma, d}(x)\right), \alpha_{W}^{-1}(\mu)\right\}$. Note that for $s \in[0, \mu]$,

$$
\sigma_{d}(s)=\alpha_{W}^{-1}(\mu) .
$$

Consider now cost $\widehat{V}_{\gamma, d}(\hat{v})=\widehat{V}_{\gamma, d}\left(f\left(x, \hat{u}_{0}\right)\right)$, where $\hat{u}_{0}$ is the first input of the near-optimal sequence $\hat{\boldsymbol{u}}_{\gamma, d}(x)$. From Assumption $3, \widehat{V}_{\gamma, d}(\hat{v}) \leq V_{\gamma, d}(\hat{v})+\eta(\epsilon, \gamma, d)$. Let $\boldsymbol{u}:=$ $\left[\hat{u}_{1}, \hat{u}_{2}, \ldots, \hat{u}_{d-1}, \overline{\boldsymbol{u}}_{1}\right]$ where $\overline{\boldsymbol{u}}_{1}:=\boldsymbol{u}_{\gamma, 1}^{*}\left(\hat{\phi}_{d}\right)$ is the optimal sequence of inputs of length 2 for state $\hat{\phi}_{d}$ with cost $V_{\gamma, 1}\left(\hat{\phi}_{d}\right)$ and $\hat{u}_{i}$ are the inputs of the near-optimal sequence. It follows from the optimality of $V_{\gamma, d}(\hat{v})$ that $V_{\gamma, d}(\hat{v}) \leq J_{\gamma, d}(\hat{v}, \boldsymbol{u}) \leq$ $J_{\gamma, d-2}\left(\hat{v},\left[\hat{u}_{1}, \ldots, \hat{u}_{d-1}\right]\right)+\gamma^{d-1} V_{\gamma, 1}\left(\phi_{d}\right)$. Therefore, according to Assumption 3,

$$
\begin{aligned}
& \widehat{V}_{\gamma, d}(\hat{v}) \\
& \leq J_{\gamma, d-2}\left(\hat{v},\left[\hat{u}_{1}, \ldots, \hat{u}_{d-1}\right]\right)+\gamma^{d-1} V_{\gamma, 1}\left(\hat{\phi}_{d}\right)+\eta(\epsilon, \gamma, d) .
\end{aligned}
$$

On the other hand, we have $\widehat{V}_{\gamma, d}(x)=$ $J_{\gamma, d}\left(x,\left[\hat{u}_{0}, \hat{u}_{1}, \ldots, \hat{u}_{d}\right]\right)=\hat{\ell}_{0}+\gamma J_{\gamma, d-1}\left(\hat{v},\left[\hat{u}_{1}, \ldots, \hat{u}_{d}\right]\right)$, which implies $J_{\gamma, d-2}\left(\hat{v},\left[\hat{u}_{1}, \ldots, \hat{u}_{d-1}\right]\right) \leq$ $J_{\gamma, d-1}\left(\hat{v},\left[\hat{u}_{1}, \ldots, \hat{u}_{d}\right]\right)=\frac{\widehat{V}_{\gamma, d}(x)-\hat{\ell}_{0}}{\gamma}$. In view of (59),

$$
\begin{aligned}
& \widehat{V}_{\gamma, d}(\hat{v})-\widehat{V}_{\gamma, d}(x) \\
& \leq J_{\gamma, d-2}\left(\hat{v},\left[\hat{u}_{1}, \ldots, \hat{u}_{d-1}\right]\right)+\gamma^{d-1} V_{\gamma, 1}\left(\hat{\phi}_{d}\right)+\eta(\epsilon, \gamma, d) \\
& -\widehat{V}_{\gamma, d}(x) \\
& \leq \frac{\widehat{V}_{\gamma, d}(x)-\hat{\ell}_{0}}{\gamma}-\widehat{V}_{\gamma, d}(x)+\gamma^{d-1} V_{\gamma, 1}\left(\hat{\phi}_{d}\right)+\eta(\epsilon, \gamma, d) \\
& \leq \frac{-\hat{\ell}_{0}+(1-\gamma) \widehat{V}_{\gamma, d}(x)+\gamma^{d} V_{\gamma, 1}\left(\hat{\phi}_{d}\right)+\gamma \eta(\epsilon, \gamma, d)}{\gamma} .
\end{aligned}
$$

From item (ii) of Assumption 1 and following the steps in the proof of Theorem 1 to obtain (30), we have

$$
W(\hat{v})-W(x) \leq \frac{1}{\gamma}\left(-\alpha_{W}(\sigma(x))+\hat{\ell}_{0}+(1-\gamma) W(x)\right) .
$$

Summing equations (60) and (61), we have

$$
\begin{aligned}
& \widehat{Y}_{\gamma, d}(\hat{v})-\widehat{Y}_{\gamma, d}(x) \\
& \leq \frac{-\alpha_{W}(\sigma(x))+(1-\gamma) \widehat{Y}_{\gamma, d}(x)+\gamma^{d} V_{\gamma, 1}\left(\hat{\phi}_{d}\right)+\gamma \eta(\epsilon, \gamma, d)}{\gamma} .
\end{aligned}
$$

Recall that $\widehat{Y}_{\gamma, d}(x) \leq \bar{\alpha}_{Y}(\sigma(x))+\eta(\epsilon, \gamma, d)$. Again, we distinguish two cases. First, consider $\bar{\alpha}_{Y}(\sigma(x)) \geq \eta(\epsilon, \gamma, d)$. From (53), $\widetilde{\alpha}_{Y}\left(\widehat{Y}_{\gamma, d}(x)\right)=\alpha_{W} \circ \bar{\alpha}_{Y}^{-1}\left(\frac{\widehat{Y}_{\gamma, d}(x)}{2}\right) \leq \alpha_{W}(\sigma(x))$, thus $-\alpha_{W}(\sigma(x)) \leq-\widetilde{\alpha}_{Y}\left(\widehat{Y}_{\gamma, d}(x)\right)$. On the other hand, from (i) from Assumption 1 and (57), $V_{\gamma, 1}\left(\hat{\phi}_{d}\right) \leq \bar{\alpha}_{V}\left(\sigma\left(\hat{\phi}_{d}\right)\right) \leq \bar{\alpha}_{V} \circ$ $\sigma_{d}\left(\widehat{Y}_{\gamma, d}(x)\right)$. Moreover, $\gamma \eta(\epsilon, \gamma, d) \leq \frac{\gamma^{2}}{2} \mu$ since $\eta(\epsilon, \gamma, d) \leq$ $\frac{\gamma}{2} \mu$. Altogether, in view of (62),

$$
\widehat{Y}_{\gamma, d}(\hat{v})-\widehat{Y}_{\gamma, d}(x) \leq \frac{-\widetilde{\alpha}_{Y}+\widehat{\Upsilon}(\cdot, \mu, \gamma, d)}{\gamma}\left(\widehat{Y}_{\gamma, d}(x)\right)
$$

holds with $\widehat{\Upsilon}(s, \mu, \gamma, d):=\left((1-\gamma) \mathbb{I}+\gamma^{d} \bar{\alpha}_{V} \circ \sigma_{d}\right)(s)+\frac{\gamma^{2}}{2} \mu$. Second, when $\bar{\alpha}_{Y}(\sigma(x)) \leq \eta(\epsilon, \gamma, d)$, it follows that $\widehat{Y}_{\gamma, d}(x) \leq$ $2 \eta(\epsilon, \gamma, d)$ in view of $\widehat{Y}_{\gamma, d}(x) \leq \bar{\alpha}_{Y}(\sigma(x))+\eta(\epsilon, \gamma, d)$. From item (i) of Assumption 1 and (57), $V_{\gamma, 1}\left(\sigma\left(\hat{\phi}_{d}\right)\right) \leq$ $\bar{\alpha}_{V} \circ \sigma_{d}\left(\widehat{Y}_{\gamma, d}(x)\right)$. Since $\widehat{Y}_{\gamma, d}(x) \leq 2 \eta(\epsilon, \gamma, d) \leq \mu$, it follows from (58) that $V_{\gamma, 1}\left(\sigma\left(\hat{\phi}_{d}\right)\right) \leq \bar{\alpha}_{V} \circ \alpha_{W}^{-1}(\mu)$. In view of (62) and $-\alpha_{W}(\sigma(x)) \leq 0$, we derive $\widehat{Y}_{\gamma, d}(\hat{v}) \leq 2 \eta(\epsilon, \gamma, d)+$ $\frac{(1-\gamma) 2 \eta(\epsilon, \gamma, d)+\gamma^{d} \bar{\alpha}_{V} \circ \alpha_{W}^{-1}(\mu)+\gamma \eta(\epsilon, \gamma, d)}{\gamma}$, that is $\widehat{Y}_{\gamma, d}(\hat{v}) \leq\left(1+\frac{\gamma}{2}\right)$ $2 \frac{\eta(\epsilon, \gamma, d)}{\gamma}+\gamma^{d-1} \bar{\alpha}_{V} \circ \alpha_{W}^{-1}(\mu)$. Therefore,

$$
\widehat{Y}_{\gamma, d}(\hat{v}) \leq \nu(\mu, \gamma, d)
$$

holds with $\nu(\mu, \gamma, d):=\left(1+\frac{\gamma}{2}\right) \mu+\gamma^{d-1} \bar{\alpha}_{V} \circ \alpha_{W}^{-1}(\mu)$. Note that $\nu(\cdot, \gamma, d)$ is class $\mathcal{K}_{\infty}$, and both $\nu(\mu, \cdot, d)$ and $\nu(\mu, \gamma, \cdot)$ can be made as small as desired by reducing $\mu$.

Let $\delta, \Delta>0$ and $^{8} \widetilde{\Delta}:=2 \bar{\alpha}_{Y}(\Delta), \widetilde{\delta}:=\left(\mathbb{I}-\frac{1}{2} \widetilde{\alpha}_{Y}\right)^{-1} \circ \alpha_{W}(\delta)$. There exists $\left(\gamma^{*}, d^{*}\right) \in(0,1) \times \mathbb{Z}_{\geq 0}$ such that for any $(\gamma, d) \in$ $\mathcal{P}_{\gamma^{*}, d^{*}}$, there exists $\epsilon^{*}>0$ such that for any $\epsilon \in\left[0, \epsilon^{*}\right)$ the following holds

$$
\begin{aligned}
\forall s \in[\widetilde{\delta}, \widetilde{\Delta}], \widehat{\Upsilon}(s, \mu, \gamma, d) & \leq\left(1-\frac{\gamma}{2}\right) \widetilde{\alpha}_{Y}(s) \\
\nu(\mu, \gamma, d) & \leq\left(\mathbb{I}-\frac{1}{2} \widetilde{\alpha}_{Y}\right)(\widetilde{\delta}) \\
1-\frac{\widetilde{\alpha}_{Y}(\mu)}{\mu} & \leq \gamma \\
\frac{2 \eta(\epsilon, \gamma, d)}{\gamma} & \leq \mu,
\end{aligned}
$$

with $\nu(\mu, \gamma, d)=\frac{1+\frac{\gamma}{2}}{\gamma} \mu+\gamma^{d-1} \bar{\alpha}_{V} \circ \alpha_{W}^{-1}(\mu)$, $\widehat{\Upsilon}(s, \mu, \gamma, d)=\left((1-\gamma) \mathbb{I}+\gamma^{d} \bar{\alpha}_{V} \circ \sigma_{d}\right)(s)+\frac{\gamma^{2}}{2} \mu$, and $\sigma_{d}(s)=\max \left\{\alpha_{W}^{-1} \circ\left(\frac{\mathbb{I}-\widetilde{\alpha}_{Y}}{\gamma}\right)^{(d)}(s), \alpha_{W}^{-1}(\mu)\right\}$. The satisfaction of (65)-(68) is not so easy to see, we therefore prove it step by step. First, note that the right hand side of (65) is bounded below by $\frac{1}{2} \widetilde{\alpha}_{Y}(\widetilde{\delta})$. On the left side, we have $\widehat{\Upsilon}(s, \mu, \gamma, d) \leq(1-\gamma) \widetilde{\Delta}+\bar{\alpha}_{V} \circ \sigma_{d}(\widetilde{\Delta})+\frac{\mu}{2}=: \psi(\mu, \gamma, d)$. By the same arguments given in the proof of Theorem 2 , we have that $\psi(\mu, \gamma, d) \rightarrow \bar{\alpha}_{V} \circ \alpha_{W}^{-1}(\mu)+\frac{\mu}{2}$ as $(\gamma, d) \rightarrow(1, \infty)$, which in turn can be made as small as desired by taking $\mu$ small. Thus, like in the proof of Theorem 2, there exist a triple $\left(\mu^{*}{ }_{(65)}, \gamma^{*}{ }_{(65)}, d^{*}{ }_{(65)}\right)$ such that for any $(\mu, \gamma, d) \in\left[0, \mu^{*}{ }_{(65)}\right) \times \mathcal{P}_{\gamma^{*}(65), d^{*}(65)}$, $\widehat{\Upsilon}(s, \mu, \gamma, d) \leq \psi(\mu, \gamma, d) \leq \frac{1}{2} \widetilde{\alpha}_{Y}(\widetilde{\delta})$ and thus (65) holds. Regarding (66), note that its right hand side is positive since $\widetilde{\delta}>0$ and $^{8} \mathbb{I}-\frac{\widetilde{\alpha}_{Y}}{2} \in \mathcal{K}_{\infty}$. We have that $\nu(\mu, \gamma, d) \leq \frac{1+\frac{\gamma}{2}}{\gamma} \mu+\bar{\alpha}_{V} \circ \alpha_{W}^{-1}(\mu)=: \psi^{\prime}(\gamma, \mu)$. Since $\frac{1+\frac{\gamma}{2}}{\gamma}$ is decreasing in $\gamma$, we have that $\psi^{\prime}$ is decreasing in $\gamma$ and can be

${ }^{8}$ As seen in the proof of Theorem $2, \mathbb{I}-\widetilde{\alpha}_{Y} \in \mathcal{K}_{\infty}$ implies $\mathbb{I}-\frac{\widetilde{\alpha}_{Y}}{2} \in \mathcal{K}_{\infty}$, thus $\widetilde{\delta}$ is well defined. 
made as small as desired by taking $\mu$ small. Thus in the same vein as for $\psi$ for (65), there exists a triple $\left(\mu^{*}{ }_{(66)}, \gamma^{*}{ }_{(66)}, d^{*}{ }_{(66)}\right)$ such that for any $(\mu, \gamma, d) \in\left[0, \mu^{*}{ }_{(66)}\right) \times \mathcal{P}_{\gamma^{*}(66)}, d^{*}{ }_{(66)}$, $\nu(\mu, \gamma, d) \leq \phi^{\prime}(\mu, \gamma) \leq\left(\mathbb{I}-\frac{1}{2} \widetilde{\alpha}_{Y}\right)(\widetilde{\delta})$ and thus (66) holds. For (67), note that $1-\frac{\widetilde{\alpha}_{Y}(\mu)}{\mu}<1$ for $\mu>0$. Thus, for each $\mu>0$, there exist $\gamma^{*}{ }_{(67)} \in(0,1)$ such that for any $\gamma \in\left(\gamma^{*}{ }_{(67)}, 1\right]$, (67) holds. For (68), since $\eta(\cdot, \gamma, d) \in \mathcal{K}_{\infty}$, $\eta(\epsilon, \gamma, d)$ can be made as small as desired by reducing $\epsilon$ in function of $(\mu, \gamma, d)$. Furthermore, for any $(\mu, \gamma, d)$, there exists $\epsilon^{*}{ }_{(68)}$ such that for any $\epsilon \in\left[0, \epsilon^{*}{ }_{(68)}\right), \frac{2 \eta(\epsilon, \gamma, d)}{\gamma} \leq \mu$. In sum, we select $\left(\mu^{*}, \gamma^{*}, d^{*}\right) \in\left(0, \min \left\{\mu_{(65)}, \mu_{(66)}\right\}\right) \times$ $\mathcal{P}_{\max \left\{\gamma^{*}{ }_{(65)}, \gamma^{*}{ }_{(66)}, \gamma^{*}(67)\right\}, \max \left\{d^{*}{ }_{(65)}, d^{*}{ }_{(66)}\right\} \text { and for each }}$ $(\gamma, d) \in \mathcal{P}_{\gamma^{*}, d^{*}}$, we take $\epsilon^{*} \in\left(0, \epsilon^{*}{ }_{(68)}\right)$. In that way (65)-(67) are satisfied. This choice of $\left(\gamma^{*}, d^{*}\right)$ is able to verify the properties mentioned beforehand. That is, inequalities (65)-(67) hold for $\mu^{*}$ and for every $\epsilon \leq \epsilon^{*}{ }_{(68)}$ chosen in function of $(\gamma, d) \in \mathcal{P}_{\gamma^{*}, d^{*}}$, so is (68). u We now follow similar lines as in the proof of Theorem 2 to prove (20). When $\bar{\alpha}_{Y}(\sigma(x)) \geq \eta(\epsilon, \gamma, d)$, (63) holds. In this case, in view of (65), $\widehat{Y}_{\gamma, d}(\hat{v})-\widehat{Y}_{\gamma, d}(x) \leq-\frac{1}{2} \widetilde{\alpha}_{Y}\left(\widehat{Y}_{\gamma, d}(x)\right)$ if $\widehat{Y}_{\gamma, d} \in[\widetilde{\delta}, \widetilde{\Delta}]$, and if $\widehat{Y}_{\gamma, d}(x) \in[0, \widetilde{\delta})$, we derive ${ }^{9}$ $\widehat{Y}_{\gamma, d}(\hat{v}) \leq \widetilde{\delta}-\frac{1}{2} \widetilde{\alpha}_{Y}(\widetilde{\delta}) \leq\left(\mathbb{I}-\frac{1}{2} \widetilde{\alpha}_{Y}\right)(\widetilde{\delta})$. For $\bar{\alpha}_{Y}(\sigma(x)) \leq \eta(\epsilon, \gamma, d)$, (64) holds. Hence, in view of (66), $\widehat{Y}_{\gamma, d}(\hat{v}) \leq \nu(\mu, \gamma, d) \leq\left(\mathbb{I}-\frac{1}{2} \widetilde{\alpha}_{Y}\right)(\widetilde{\delta})$. By the same line of reasoning as in the proof of Theorem 2, we deduce that there exist $\hat{\beta} \in \mathcal{K} \mathcal{L}$, which is uniform in $\gamma$ and $d$, such that any solution $\hat{\phi}$ to (19) initialized at $\sigma(x) \leq \Delta$ and any $k \in \mathbb{Z}_{\geq 0}$,

$$
\widehat{Y}_{\gamma, d}(\hat{\phi}(k, x)) \leq \max \left\{\hat{\beta}\left(\widehat{Y}_{\gamma, d}(x), k\right),\left(\mathbb{I}-\frac{1}{2} \widetilde{\alpha}_{Y}\right)(\widetilde{\delta})\right\} .
$$

Finally, recall $\underline{\alpha}_{Y}(\sigma(x)) \leq \widehat{Y}_{\gamma, d}(x) \leq \bar{\alpha}_{Y}(\sigma(x))+\eta(\epsilon, \gamma, d) \leq$ $2 \max \left\{\bar{\alpha}_{Y}(\sigma(x)), \eta(\epsilon, \gamma, d)\right\}$ and $\widetilde{\delta}=\left(\mathbb{I}-\frac{1}{2} \widetilde{\alpha}_{Y}\right)^{-1} \circ \underline{\alpha}_{Y}(\delta)$. Then, since $\hat{\beta} \in \mathcal{K} \mathcal{L}$, we derive

$$
\begin{aligned}
\sigma(\hat{\phi}(k, x)) \leq \max \{ & \underline{\alpha}_{Y}^{-1}\left(\hat{\beta}\left(2 \bar{\alpha}_{Y}(\sigma(x)), k\right)\right), \\
& \left.\underline{\alpha}_{Y}^{-1}\left(\hat{\beta}\left(2 \eta\left(\epsilon^{*}, \gamma, d\right), 0\right)\right), \delta\right\} .
\end{aligned}
$$

Let $\epsilon^{*} \in\left[0, \epsilon^{*}{ }_{(68)}\right)$ be such that $\underline{\alpha}_{Y}^{-1}\left(\hat{\beta}\left(2 \eta\left(\epsilon^{*}, \gamma, d\right), 0\right)\right) \leq \delta$, we obtain

$$
\sigma(\hat{\phi}(k, x)) \leq \max \left\{\underline{\alpha}_{Y}^{-1}\left(\hat{\beta}\left(2 \bar{\alpha}_{Y}(\sigma(x)), k\right)\right), \delta\right\} .
$$

Thus (20) holds with $\beta(s, k)=\underline{\alpha}_{Y}^{-1}\left(\hat{\beta}\left(2 \bar{\alpha}_{Y}(s), k\right)\right)$.

We now prove the last part of Theorem 4 . We have already noted the existence of a pair $\left(\gamma^{*}, d^{*}\right)$ such that inequalities (65)-(67) hold for any $\gamma \in\left(\gamma^{*}, 1\right]$ and $d \in\left(d^{*}, \infty\right]$ for fixed $\mu$. Assume, without loss of generality, that $2 \frac{\left.\eta\left(\epsilon^{*}(68)\right), \gamma^{*}, d^{*}\right)}{\gamma^{*}} \leq \mu$. It follows that $\left.\eta(\epsilon, \gamma, d) \leq \eta\left(\epsilon^{*}{ }_{(68)}\right), \gamma^{*}, d^{*}\right)$, as $\eta$ is assumed here to be non-decreasing in $\gamma$ and $d$, and is of class $\mathcal{K}_{\infty}$ in $\epsilon$. Since $\gamma^{*} \leq \gamma, \frac{1}{\gamma} \leq \frac{1}{\gamma^{*}}$. Hence, $2 \frac{\eta(\epsilon, \gamma, d)}{\gamma} \leq \mu$. Therefore, the remaining inequality (68) is verified for any

\footnotetext{
${ }^{9}$ This holds with the same reasoning as (37) in the proof of Theorem 2, with the following rectification. In (37), we have utilized $\Upsilon(\cdot, \gamma, d)-(1-\gamma) \mathbb{I} \in \mathcal{K}_{\infty}$ to show that $\gamma \mathbb{I}-\alpha_{W} \circ \bar{\alpha}_{Y}^{-1}+\Upsilon(\cdot, \gamma, d) \in \mathcal{K}_{\infty}$. Here, it suffices to note that $\widehat{\Upsilon}(\cdot, \mu, \gamma, d)-(1-\gamma) \mathbb{I}$ is non-decreasing, henceforth by following the same steps as in (37) the mentioned bound is found.
}

$(\epsilon, \gamma, d) \in\left[0, \epsilon^{*}\right) \times \mathcal{P}_{\gamma^{*}, d^{*}}$ and (70) holds. All that is left to find is $\epsilon^{*} \leq \epsilon^{*}{ }_{(68)}$ such that $\underline{\alpha}_{Y}^{-1}\left(\hat{\beta}\left(2\left(\epsilon^{*}, \gamma^{*}, d^{*}\right), 0\right)\right) \leq \delta$, hence $\underline{\alpha}_{Y}^{-1}(\hat{\beta}(2 \eta(\epsilon, \gamma, d), 0)) \leq \delta$ and the proof is complete.

\section{K. Proof of Lemma 4}

Let $s \in \mathbb{R}_{\geq 0}, \gamma \in(0,1], d \in \mathbb{Z}_{>0} \cup\{\infty\}, \delta, \Delta>0$ and recall the definitions for $\Upsilon, \widehat{\Upsilon}$ and respective $\widetilde{\alpha}_{Y}$ from the proofs of Theorem 1 and Theorem 4. It follows that $\Upsilon(s, \gamma, d)=$ $(1-\gamma) s+\gamma^{d} \bar{\alpha}_{V} \circ \alpha_{W}^{-1}\left(\frac{\mathbb{I}-\alpha_{W} \circ\left(\bar{\alpha}_{V}+\bar{\alpha}_{W}\right)^{-1}}{\gamma}\right)^{(d)}(s) \leq(1-$ $\gamma) s+\gamma^{d} \bar{\alpha}_{V} \circ \alpha_{W}^{-1}\left(\frac{\mathbb{I}-\alpha_{W} \circ\left(\bar{\alpha}_{V}+\bar{\alpha}_{W}\right)^{-1} \circ \frac{\mathbb{I}}{2}}{\gamma}\right)^{(d)}(s)$. On the other hand, for any $\mu \geq 0$, we have that $\left(\frac{\mathbb{I}-\alpha_{W} \circ\left(\bar{\alpha}_{V}+\bar{\alpha}_{W}\right)^{-1} \circ \frac{\mathbb{I}}{2}}{\gamma}\right)^{(d)} \leq$ $\max \left\{\left(\frac{\mathbb{I}-\alpha_{W} \circ\left(\bar{\alpha}_{V}+\bar{\alpha}_{W}\right)^{-1} \circ \frac{\mathbb{I}}{2}}{\gamma}\right)^{(d)}, \mu\right\}$. Therefore $\Upsilon(s, \gamma, d) \leq$ $\widehat{\Upsilon}(s, \mu, \gamma, d)-\frac{\mu^{2}}{2} \leq \widehat{\Upsilon}(s, \mu, \gamma, d)$. On the other hand, we have $\widetilde{\alpha}_{Y}=\alpha_{W} \circ \bar{\alpha}_{Y}^{-1}$ in Theorem 1 and $\widetilde{\alpha}_{Y}=\alpha_{W} \circ \bar{\alpha}_{Y}^{-1} \circ \frac{\mathbb{I}}{2}$. Clearly, when (65) holds for pair $(\delta, \Delta)$ for all $(\mu, \gamma, d) \in\left[0, \mu^{*}\right) \times$ $\left(\gamma^{*}, 1\right] \times\left(d^{*}, \infty\right]$, it follows that $\Upsilon(s, \gamma, d) \leq \widehat{\Upsilon}(s, \mu, \gamma, d$, $\left(1-\frac{\gamma}{2}\right) \alpha_{W} \circ \bar{\alpha}_{Y}^{-1} \circ \frac{\mathbb{I}}{2} \leq\left(1-\frac{\gamma}{2}\right) \alpha_{W} \circ \bar{\alpha}_{Y}^{-1}(s)$, thus equation (34) holds for all $(\gamma, d) \in\left(\gamma^{*}, 1\right] \times\left(d^{*}, \infty\right]$ and the proof is complete.

\section{Proof of Lemma 4}

The proof immediately follows from Assumption 3 and item (i) of Theorem 3, which holds from Assumption 1. In particular $\widehat{V}_{\gamma, d}(x)-\eta(\epsilon, \gamma, d) \leq V_{\gamma, d}(x) \leq V_{\gamma, \infty}(x) \leq$ $V_{\gamma, d}(x)+\gamma^{d} v_{\gamma, d}(x) \leq \widehat{V}_{\gamma, d}(x)+\gamma^{d} v_{\gamma, d}(x)$.

\section{CONCLUSION}

We have analyzed the stability of nonlinear discrete-time systems controlled by a sequence of inputs that minimizes a discounted finite-horizon cost and is implemented in a receding-horizon fashion. In general, uniform semiglobal practical stability is ensured under suitable stabilizability and detectability conditions. Under additional assumptions, semiglobal asymptotic stability and uniform global exponential stability properties are guaranteed. The Lyapunov function used to prove stability is shown to be continuous under extra assumptions, hence endowing stability with some nominal robustness. We compared our results with previous works of the literature on undiscounted finite-horizon and discounted infinite-horizon optimal control, respectively. We have identified conditions under which the bounds we provide on discount factor and on horizon length are less conservative than those in [21] and [6]. Furthermore, we provide new relationships between the optimal value functions of the discounted, undiscounted, infinite-horizon, finite-horizon costs, which differ from those typically found in the (approximate) dynamic programming literature. Finally, we have analyzed stability when the available inputs are only near-optimal, for which an illustrative example was provided.

It will be interesting in future work to extend these results to the stochastic case, for which dynamic programming algorithms are available [1], in particular because of the relationship between infinite-horizon costs and finite-horizon ones [18]. 


\section{APPENDIX}

The next Lyapunov properties are used in the proof of Theorem 1 .

Theorem 5: Suppose Assumption 1 is verified. Functions $\alpha_{Y}, \bar{\alpha}_{Y}, \alpha_{Y} \in \mathcal{K}_{\infty}$ from Theorem 1 are such that, for any $\gamma \in$ $(0,1], d \in \mathbb{Z}_{>0}, k \in\{0, \ldots, d\}$, and $Y_{\gamma, d-k}:=V_{\gamma, d-k}+W$ is such that the following holds.

(i) For any $x \in \mathbb{R}^{n}$,

$$
\underline{\alpha}_{Y}(\sigma(x)) \leq Y_{\gamma, d-k}(x) \leq \bar{\alpha}_{Y}(\sigma(x)) .
$$

(ii) For any $x \in \mathbb{R}^{n}$,

$$
\begin{aligned}
& Y_{\gamma, d-(k+1)}\left(\phi_{k+1}^{*}\right)-Y_{\gamma, d-k}\left(\phi_{k}^{*}\right) \\
& \leq \frac{1}{\gamma}\left(-\alpha_{Y}\left(\sigma\left(\phi_{k}^{*}\right)\right)+(1-\gamma) Y_{\gamma, d-k}\left(\phi_{k}^{*}\right)\right),
\end{aligned}
$$

where $\phi_{k+1}^{*} \in F_{\gamma, d-k}^{*}\left(\phi_{k}^{*}\right)$ and $\phi_{0}^{*}=x$.

Proof of Theorem 5: Let $\gamma \in(0,1], d \in \mathbb{Z}_{>0}, k \in$ $\{0, \ldots, d\}, x \in \mathbb{R}^{n}$. There exists $\left[u_{0}^{*}, u_{1}^{*}, \ldots, u_{d}^{*}\right]=\boldsymbol{u}_{\gamma, d}^{*}(x)$ where $\boldsymbol{u}_{\gamma, d}^{*}(x)$ is an optimal input sequence for system (1) with cost (2) according to SA.

Define $\ell_{0}^{*}=\ell\left(\phi_{0}^{*}, u_{0}^{*}\right)$ and let $\boldsymbol{u}^{\prime}$ be the sequence of length $d$ such that $\boldsymbol{u}_{\gamma, d}^{*}(x)=\left[u_{0}^{*}, \boldsymbol{u}^{\prime}\right]$. From the definition of $V_{\gamma, d}$ in (3) and cost (2), $V_{\gamma, d}(x)=J_{\gamma, 0}\left(x, u_{0}^{*}\right)+$ $\gamma J_{\gamma, d-1}\left(f\left(x, u_{0}^{*}\right), \boldsymbol{u}^{\prime}\right)=\ell_{0}^{*}+\gamma J_{\gamma, d-1}\left(\phi_{1}^{*}, \boldsymbol{u}^{\prime}\right)$ By definition of $V_{\gamma, d}(x)$ and $\boldsymbol{u}^{\prime}, V_{\gamma, d-1}\left(\phi_{1}^{*}\right)=J_{\gamma, d-1}\left(f\left(x, u_{0}^{*}\right), \boldsymbol{u}^{\prime}\right)$. Hence, $V_{\gamma, d}(x)=\ell_{0}^{*}+\gamma V_{\gamma, d-1}\left(\phi_{1}^{*}\right)$. Let $\ell_{k}^{*}=\ell\left(\phi_{k}^{*}, u_{k}^{*}\right)$. Remark that, when $k<d$ and by iteration, $V_{\gamma, d-k}\left(\phi_{k}^{*}\right)=J_{\gamma, 0}\left(\phi_{k}^{*}, u_{k}^{*}\right)+$ $\gamma J_{\gamma, d-(k+1)}\left(f\left(\phi_{k}^{*}, u_{k}^{*}\right), \boldsymbol{u}_{\gamma, d-(k+1)}^{*}\left(f\left(\phi_{k}^{*}, u_{k}^{*}\right)\right)\right)=\ell_{k}^{*}+$ $\gamma \min _{\boldsymbol{u}} J_{\gamma, d-(k+1)}\left(\phi_{k+1}^{*}, \boldsymbol{u}\right)$, hence

$$
V_{\gamma, d-k}\left(\phi_{k}^{*}\right)=\ell_{k}^{*}+\gamma V_{\gamma, d-(k+1)}\left(\phi_{k+1}^{*}\right) .
$$

Notice that for $k=d, V_{\gamma, 0}\left(\phi_{d}^{*}\right)=\ell_{d}^{*}$ since $J_{\gamma, 0}(x, u)=$ $\ell(x, u)$. Since stage cost $\ell$ is nonnegative and item (i) of Assumption 1 holds for any $d$, it follows from (74)

$$
\ell_{k}^{*} \leq V_{\gamma, d-k}\left(\phi_{k}^{*}\right) \leq \bar{\alpha}_{V}\left(\sigma\left(\phi_{k}^{*}\right)\right) .
$$

Furthermore, using Bellman equation,

$$
\begin{aligned}
& V_{\gamma, d-(k+1)}\left(\phi_{k+1}^{*}\right)-V_{\gamma, d-k}\left(\phi_{k}^{*}\right) \\
& \quad \leq V_{\gamma, d-(k+1)}\left(\phi_{k+1}^{*}\right)-\ell_{k}^{*}-\gamma V_{\gamma, d-(k+1)}\left(\phi_{k+1}^{*}\right) \\
& \quad \leq-\ell_{k}^{*}+(1-\gamma) V_{\gamma, d-(k+1)}\left(\phi_{k+1}^{*}\right) .
\end{aligned}
$$

It follows from (74) that $V_{\gamma, d-(k+1)}\left(\phi_{k+1}^{*}\right)=\frac{1}{\gamma}\left(V_{\gamma, d-k}\left(\phi_{k}^{*}\right)-\right.$ $\left.\ell_{k}^{*}\right)$, hence

$$
\begin{aligned}
V_{\gamma, d-(k+1)} & \left(\phi_{k+1}^{*}\right)-V_{\gamma, d-k}\left(\phi_{k}^{*}\right) \\
\leq & \frac{1}{\gamma}\left(-\ell_{k}^{*}+(1-\gamma) V_{\gamma, d-k}\left(\phi_{k}^{*}\right)\right) .
\end{aligned}
$$

On the other hand, in view of item (ii) of Assumption 1, $W\left(f\left(\phi_{k}^{*}, u_{k}^{*}\right)\right)-W\left(\phi_{k}^{*}\right) \leq-\alpha_{W}\left(\sigma\left(\phi_{k}^{*}\right)\right)+\ell\left(\phi_{k}^{*}, u_{k}^{*}\right)$. Since $\phi_{k+1}^{*}=f\left(\phi_{k}^{*}, u_{k}^{*}\right)$ and $\ell_{k}^{*}=\ell\left(\phi_{k}^{*}, u_{k}^{*}\right), W\left(\phi_{k+1}^{*}\right)-W\left(\phi_{k}^{*}\right) \leq$ $-\alpha_{W}\left(\sigma\left(\phi_{k}^{*}\right)\right)+\ell_{k}^{*}$. Furthermore, since $\gamma \leq 1, \gamma W\left(\phi_{k+1}^{*}\right)-$ $W\left(\phi_{k}^{*}\right) \leq-\alpha_{W}\left(\sigma\left(\phi_{k}^{*}\right)\right)+\ell_{k}^{*}$, hence $W\left(\phi_{k+1}^{*}\right)-W\left(\phi_{k}^{*}\right)-$ $\frac{1-\gamma}{\gamma} W\left(\phi_{k}^{*}\right) \leq-\frac{\alpha_{W}\left(\sigma\left(\phi_{k}^{*}\right)\right)}{\gamma}+\frac{\ell_{k}^{*}}{\gamma}$ as in (30). Therefore,

$$
\begin{aligned}
& W\left(\phi_{k+1}^{*}\right)-W\left(\phi_{k}^{*}\right) \\
& \quad \leq \frac{1}{\gamma}\left(-\alpha_{W}\left(\sigma\left(\phi_{k}^{*}\right)\right)+\ell_{k}^{*}+(1-\gamma) W(x)\right)
\end{aligned}
$$

We define $Y_{\gamma, k}:=V_{\gamma, k}+W$. In view of (77) and (78),

$$
\begin{aligned}
& Y_{\gamma, d-(k+1)}\left(\phi_{k+1}^{*}\right)-Y_{\gamma, d-k}\left(\phi_{k}^{*}\right) \\
& \leq \frac{1}{\gamma}\left(-\ell_{k}^{*}+(1-\gamma) V_{\gamma, d-k}\left(\phi_{k}^{*}\right)-\alpha_{W}\left(\sigma\left(\phi_{k}^{*}\right)\right)+\ell_{k}^{*}\right. \\
& \left.\quad \quad+(1-\gamma) W\left(\phi_{k}^{*}\right)\right) \\
& =\frac{1}{\gamma}\left(-\alpha_{W}\left(\sigma\left(\phi_{k}^{*}\right)\right)+(1-\gamma) Y_{\gamma, d-k}\left(\phi_{k}^{*}\right)\right) .
\end{aligned}
$$

Thus item (ii) is verified with $\alpha_{Y}=\alpha_{W} \in \mathcal{K}_{\infty}$.

On the other hand, item (i) of Theorem 5 follows by noting that item (i) of Theorem 1 holds for any $x \in \mathbb{R}^{n}, d \in \mathbb{Z}_{>0}$ and $\gamma \in(0,1]$

\section{REFERENCES}

[1] D. P. Bertsekas. Dynamic Programming and Optimal Control, volume 2. Athena Scientific, Nashua, USA, 4th edition, 2012.

[2] L. Buşoniu, D. Ernst, B. D. Schutter, and R. Babuška. Approximate dynamic programming with a fuzzy parameterization. Automatica, 46(5):804 - 814, 2010 .

[3] D. Ernst, P. Geurts, and L. Wehenkel. Tree-based batch mode reinforcement learning. Journal of Machine Learning Research, 6:503556, 2005.

[4] V. Gaitsgory, L. Grüne, M. Höger, C. M. Kellett, and S. R. Weller Stabilization of strictly dissipative discrete time systems with discounted optimal control. Automatica, 93:311 - 320, 2018.

[5] M. Granzotto, R. Postoyan, L. Buşoniu, D. Nešić, and J. Daafouz. Stability analysis of discrete-time finite-horizon discounted optimal control. In IEEE Conference on Decision and Control, pages 23222327, Miami, USA, 2018.

[6] G. Grimm, M. J. Messina, S. E. Tuna, and A. R. Teel. Model predictive control: for want of a local control Lyapunov function, all is not lost. IEEE Transactions on Automatic Control, 50(5):546-558, 2005.

[7] L. Grüne. Computing stability and performance bounds for unconstrained NMPC schemes. In IEEE Conference on Decision and Control, pages 1263-1268, New Orleans, USA, 2007.

[8] L. Grüne and A. Rantzer. On the infinite horizon performance of receding horizon controllers. IEEE Transactions on Automatic Control, 53(9):2100 2111, 2008.

[9] L. Grüne, W. Semmler, and M. Stieler. Using nonlinear model predictive control for dynamic decision problems in economics. Journal of Economic Dynamics and Control, 60:112-133, 2015.

[10] A. Heydari. Stability analysis of optimal adaptive control using value iteration with approximation errors. IEEE Transactions on Automatic Control, 63(9):3119-3126, 2018.

[11] J.-F. Hren and R. Munos. Optimistic planning of deterministic systems In European Workshop on Reinforcement Learning, pages 151-164, Villeneuve d'Ascq, France, 2008. Springer.

[12] Z.-P. Jiang and Y. Wang. Input-to-state stability for discrete-time nonlinear systems. Automatica, 37(6):857-869, 2001.

[13] R.E. Kalman. Contributions to the theory of optimal control. Bol. soc. mat. mexicana, 5(2):102-119, 1960.

[14] S. Keerthi and E. Gilbert. An existence theorem for discrete-time infinitehorizon optimal control problems. IEEE Transactions on Automatic Control, 30(9):907-909, 1985.

[15] S. Keerthi and E. G Gilbert. Optimal infinite-horizon feedback laws for a general class of constrained discrete-time systems: Stability and moving-horizon approximations. Journal of optimization theory and applications, 57(2):265-293, 1988.

[16] C. M. Kellett and A. R. Teel. On the robustness of $\mathcal{K} \mathcal{L}$-stability for difference inclusions: Smooth discrete-time Lyapunov functions. SIAM Journal on Control and Optimization, 44(3):777-800, 2005.

[17] P.-J. Meyer. Invariance and symbolic control of cooperative systems for temperature regulation in intelligent buildings. $\mathrm{PhD}$ thesis, Université Grenoble Alpes, France, 2015. See Chapter 3.

[18] S. Meyn, R. L. Tweedie, and P. W. Glynn. Markov Chains and Stochastic Stability. Cambridge University Press, New York, 2nd edition, 2009.

[19] R. Munos and C. Szepesvári. Finite time bounds for fitted value iteration. Journal of Machine Learning Research, 9:815-857, 2008.

[20] M. A. Müller and L. Grüne. On the relation between dissipativity and discounted dissipativity. In IEEE 56th Annual Conference on Decision and Control (CDC), pages 5570-5575, Melbourne, AU, 2017. 
[21] R. Postoyan, L. Buşoniu, D. Nešić, and J. Daafouz. Stability analysis of discrete-time infinite-horizon optimal control with discounted cost. IEEE Transactions on Automatic Control, 62(6):2736-2749, 2017.

[22] R. T. Rockafellar and R. J.-B. Wets. Variational analysis, volume 317. Springer Science \& Business Media, 2009.

[23] R. S. Sutton and A. G. Barto. Reinforcement Learning: An Introduction. MIT Press, Cambridge, USA, 2nd edition, 2017.

[24] S. E. Tuna, M. J. Messina, and A. R. Teel. Shorter horizons for model predictive control. In American Control Conference, pages 863-868, Minneapolis, USA, 2006.

[25] C. J. C. H. Watkins and P. Dayan. Q-learning. Machine Learning, 8:279-292, 1992.

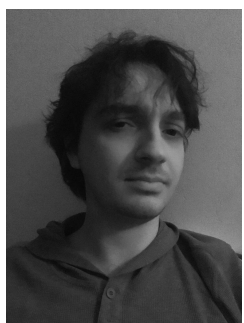

Mathieu Granzotto received his engineering degree in Control and Automation from UFSC (Brazil) in 2016. In 2019, he received his Ph.D. in Control Theory from Université de Lorraine (France), where he is a Temporary Research and Teaching Attaché at the "Centre de Recherche en Automatique de Nancy" (France). His research interests include non-linear systems, optimal control and dynamic programming.

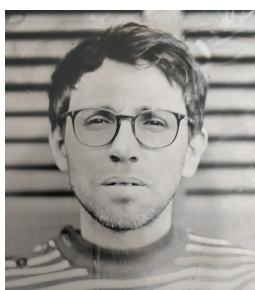

Romain Postoyan received the M.Sc. degree in Electrical and Control Engineering from ENSEEIHT (France) in 2005. He obtained the M.Sc. by Research in Control Theory \& Application from Coventry University (United Kingdom) in 2006 and the Ph.D in Control Theory from Université Paris-Sud (France) in 2009. In 2010, he was a research assistant at the University of Melbourne (Australia). Since 2011, he is a CNRS researcher at the "Centre de Recherche en Automatique de Nancy" (France). He served/serves as an Associate Editor for the journals: Automatica, IEEE Control Systems Letters and IMA Journal of Mathematical Control and Information

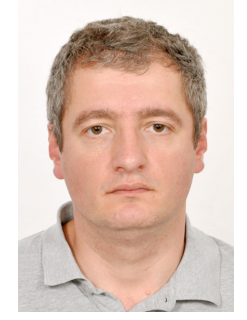

Lucian Busoniu received his $\mathrm{Ph}$.D. degree cum laude from the Delft University of Technology, the Netherlands, in 2009. He is a professor with the Department of Automation at the Technical University of ClujNapoca, where he leads the group on Robotics and Nonlinear Control. He has previously held research positions in the Netherlands and in France. He serves on the editorial board of the Elsevier journal Engineering Applications of Artificial Intelligence. His research interests include nonlinear optimal control using artificial intelligence and reinforcement learning techniques, robotics, and multiagent systems. His publications include among others several influential review articles and a book on reinforcement learning.

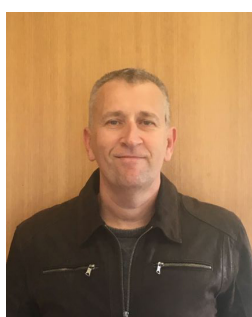

Dragan Nešić is a Professor at the Department of Electrical and Electronic Engineering (DEEE) at The University of Melbourne, Australia. He currently serves as Associate Dean Research at the Melbourne School of Engineering. He received his Bachelor of Engineering (BE) degree in Mechanical Engineering from The University of Belgrade, Serbia (1990), and his Ph.D. degree from Systems Engineering, RSISE, Australian National University, Canberra, Australia (1997). His research interests include networked control systems, reset systems, extremum seeking control, hybrid control systems, event-triggered control, security and privacy in cyber-physical systems, and so on. He published more than 400 peer reviewed journal/conference papers in top outlets in his field. He is a Fellow of the Institute of Electrical and Electronic Engineers (IEEE, 2006) and Fellow of the International Federation for Automatic Control (IFAC, 2019) $\mathrm{He}$ was awarded Doctorate Honoris Causa by the University of Lorraine, France (2019) and Humboldt Research Award (2020) by the Alexander von Humboldt Foundation. He was also awarded a Future Fellowship (2010-2014) and Australian Professorial Fellowship (2004-2009) by the Australian Research Council (ARC), as well as a Humboldt Research Fellowship (2003-2004). He was invited to serve as a Distinguished Lecturer of the Control Systems Society (CSS), IEEE (2008-2012). He was a co-recipient of the George S. Axelby Outstanding Paper Award for the Best Paper in IEEE Transactions on Automatic Control (2018). He served as an Associate Editor for the journals Automatica (2003-2015), IEEE Transactions on Automatic Control (20042008), Systems and Control Letters (2001-2010), European Journal of Control (2007-2011) and as a General Co-Chair of IEEE Conference on Decision and Control (CDC), Melbourne (2017). He currently serves as an Associate Editor for the IEEE Transactions on Control of Network Systems (since 2016). He also served as a Member of the Board of Governors, Control Systems Society (CSS), IEEE (2011-2016).

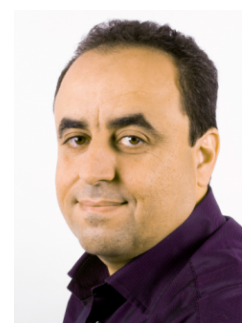

Jamal Daafouz is a Full Professor at University de Lorraine (France) and researcher at CRAN - CNRS In 1994, he received the Dipl.Ing. degree from INSA Toulouse. He prepared his Ph.D. at LAAS-CNRS Toulouse and he received the Ph.D. in Automatic Control from INSA Toulouse, in 1997. He also received the "Habilitation à Diriger des Recherches" from INPL (University de Lorraine), Nancy, in 2005.

His research interests include analysis, observation and control of uncertain systems, switched systems, hybrid systems, delay and networked systems with a particular interest for convex based optimisation methods.

In 2010, Jamal Daafouz was appointed as a junior member of the Institut Universitaire de France (IUF). He served as an associate editor of the following journals: Automatica, IEEE Transactions on Automatic Control, European Journal of Control and Non linear Analysis and Hybrid Systems. He is senior editor of the journal IEEE Control Systems Letters. 